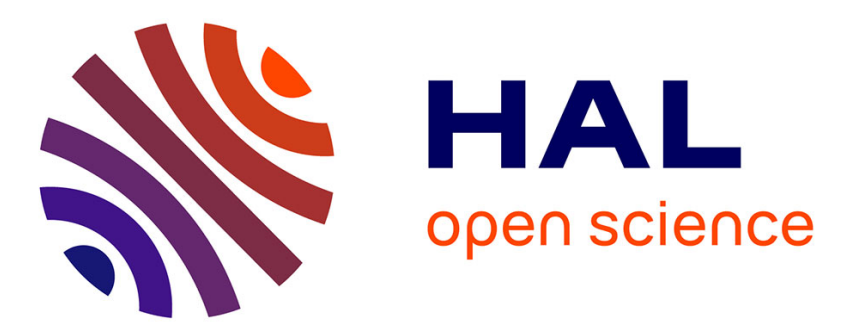

\title{
A highly anisotropic nonlinear elasticity model for vesicles I. Eulerian formulation, rigidity estimates and vanishing energy limit
}

Benoit Merlet

\section{- To cite this version:}

Benoit Merlet. A highly anisotropic nonlinear elasticity model for vesicles I. Eulerian formulation, rigidity estimates and vanishing energy limit. Archive for Rational Mechanics and Analysis, 2015, 217 (2), pp.651-680. 10.1007/s00205-014-0839-5 . hal-00848547v5

HAL Id: hal-00848547

https://hal.science/hal-00848547v5

Submitted on 2 Sep 2014

HAL is a multi-disciplinary open access archive for the deposit and dissemination of scientific research documents, whether they are published or not. The documents may come from teaching and research institutions in France or abroad, or from public or private research centers.
L'archive ouverte pluridisciplinaire $\mathbf{H A L}$, est destinée au dépôt et à la diffusion de documents scientifiques de niveau recherche, publiés ou non, émanant des établissements d'enseignement et de recherche français ou étrangers, des laboratoires publics ou privés. 


\title{
A highly anisotropic nonlinear elasticity model for vesicles I. Eulerian formulation, rigidity estimates and vanishing energy limit
}

the date of receipt and acceptance should be inserted later

\begin{abstract}
We propose a nonlinear elasticity model for vesicle membranes which is an Eulerian version of a model introduced by Pantz and Trabelsi. We describe the limit behavior of sequences of configurations whose energy goes to 0 in a fixed domain. The material is highly anisotropic and the analysis is based on some rigidity estimates adapted to this anisotropy. The main part of the paper is devoted to these estimates and to some of their consequences. The strongest form of these estimates are used in a second article to derive the thin-shell limit bending theory of the model.
\end{abstract}

Keywords Calculus of Variation - Helfrich functional - Willmore functional · Rigidity estimates · Non-linear elasticity $\cdot$ Lipid bilayers

Mathematics Subject Classification (2000) 49Q10 - 74B20 - 74K25 · 74K25

\section{Introduction}

In an aqueous environment, the components of biological or artificial vesicles selfassemble spontaneously to form large structures. Usually these components are phospholipid molecules with a hydrophilic head and two hydrophobic hydrocarbon chains. This variation of the solubility along the molecules drives the aggregation process. To lower their energy, the phospholipids form small spheres called micelles, with heads pointing towards the surrounding aqueous medium and tails pointing towards the center. They also aggregate to form large membranes made of two mono-molecular layers with all hydrophobic tails pointing toward the interior - see Figure 1.1. Because open sheet configurations would involve a huge edge energy, the bilayers form closed encapsulating structures. The enclosed volume and the bilayer membrane are called a vesicle. The composition of the fluid

Benoît Merlet

Centre de Mathématiques Appliquées (CMAP), Ecole Polytechnique, 91128 Palaiseau, France.

E-mail: merlet@cmap.polytechnique.fr 
inside a vesicle may differ from that of the surrounding medium. For this reason, vesicles play a crucial role in the organization of substances in living cells.

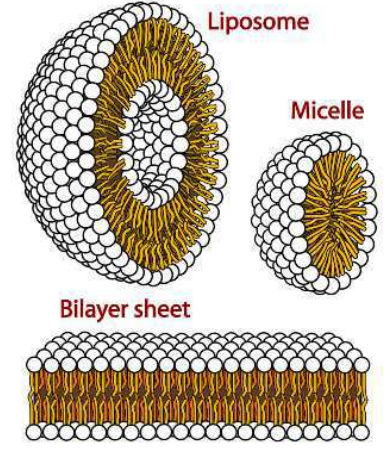

Fig. 1.1 Main phospholipid structures.

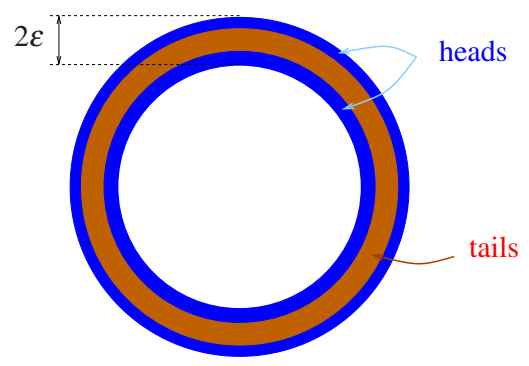

Fig. 1.2 A minimizing configuration for the model of Peletier and Röger

The vesicle membranes are a few nanometer thick whereas their size can reach the order of tenth of micrometers. In such a situation we can consider that the size of the vesicle is large with respect to the thickness of its membrane $(\operatorname{diam}(S) \gg 2 \varepsilon)$ and it is tempting to model the membrane as a surface $\Sigma=\partial O$ and to define its free energy as a function of its geometry. This point of view has been introduced by Canham [3] and Helfrich [7] — see also the review paper by Seifert [16] for a description of such models and comparisons to experiments. Vesicle membranes do not behave as other interfaces as their shapes are well described by the optimization of a bending energy and not by surface-tension theories. In the seminal paper [7], Helfrich considers an inextensional membrane represented by the surface $\Sigma$ whose elastic energy is given by the integral over $\Sigma$ of a second order polynomial function of its principal curvatures. This assumption includes the invariance of the stored energy function with respect to rotations in the tangent plane to the surface $\Sigma$. Under these conditions, he deduces the general form of the free energy of a membrane:

$$
\mathscr{W}_{H e l}(\Sigma)=\kappa_{1} \int_{\Sigma}|h-\mu|^{2}+\kappa_{2} \int_{\Sigma} K+\kappa_{3} \mathscr{H}^{2}(\Sigma) .
$$

In this formula $h$ denotes the scalar mean curvature of $\Sigma$ and $K$ denotes its Gauss curvature. These quantities are derived from the second fundamental form of $\Sigma$ which is defined as $I I=-\nabla_{\Sigma} n$ where $n$ is the outward unit normal to $\Sigma=\partial O$. With this notation, $K:=\operatorname{det} I I$ and $h:=\operatorname{Tr} I I=-\nabla_{\Sigma} \cdot n-$ notice that the sign of the scalar mean curvature depends on the choice of the orientation on $\Sigma$. Thanks to the Gauss-Bonnet formula, the second term in the definition of $\mathscr{W}_{\mathrm{Hel}}(\Sigma)$ only depends on the genus of $\Sigma$ and by assumption, the last term is a constant. Hence as soon as we only care about energy fluctuations it is equivalent to consider the energy

$$
\mathscr{W}_{\mu}(\Sigma)=\int_{\Sigma}|h-\mu|^{2} .
$$


The parameter $\mu \in \mathbf{R}$ accounts for the spontaneous curvature of the membrane which may arise for instance from differences between the properties of the environment on both sides of the membrane. When $\mu=0$, the energy simplifies to the Willmore functional

$$
\mathscr{W}(\Sigma)=\int_{\Sigma}|h|^{2}
$$

Our purpose is to derive rigorously the Helfrich energy $\mathscr{W}_{\mu}$ as a limit as $\varepsilon$ goes to 0 of a 3-D nonlinear elasticity theory for vesicle membranes with small but positive thickness $2 \varepsilon>0$.

Our work follows other attempts in this direction. Let us mention a very interesting model involving thick membranes introduced by Peletier and Röger in [12]. Their model is based on the description of the location of the tails and the heads of the lipid molecules thanks to density functions - see Figure 1.2. The Willmore energy appears as a second order term in the $\Gamma$-limit expansion of the family of energies that they consider.

The Helfrich energy also arises as the $\Gamma$-limit of some phase field models, see e.g. $[2,9]$. Such models are used to approximate the Willmore energy in image processing and our work may appear as an alternative in this field.

One can already find formal derivations of the energy of vesicle membranes from 3-D elasticity theory $[15,4]$. In these papers it is either assumed that the lipid molecules are rigid rods, or that the deformation of the material varies linearly in the out-of-plane direction. The model proposed below is also similar to a model proposed by Turner and Joanny [17] for diblock copolymer chains.

Depending on its composition, the composition of the aqueous solution and the temperature, the membrane of the vesicle displays a variety of qualitatively distinct mechanical properties. Indeed, the lipids in the bilayer can be organized in different ways and form different phases, see $e$.g. the phase diagram in [16] p12. One can find solid phases, gel phases and liquid phases. Let us mention the existence of models for 2D membranes with phase coexistence $[14,5]$ and of a model for 2D membranes in gel phase which includes the local orientation of the lipids [1]. In contrast, in the present articles we consider thick membranes in liquid phase. In this case the membrane can be seen as a fluid of rods where at the microscopic level, each lipid molecule is modeled by an elastic rod. The absence of rigidity in the directions tangent to the membrane surface leads us to consider an anisotropic elasticity theory which is degenerate in these directions.

Our choice of a continuum mechanics description is disputable. Indeed, since the thickness of a vesicle membrane is of the same order as the length of the molecules that make it up, we believe that an accurate model should be based on quantum mechanics or, at least, on a discrete description of the material. The finite thickness model for vesicle membranes is relevant as an intermediate description between a two-dimensional bending theory and Molecular Dynamics simulations. Our model is also oblivious to various phenomenons such as phase transitions in the membrane, fluid flows or the possible presence of a cytoskeleton strengthening the membrane. 
1.1 A highly anisotropic model in nonlinear elasticity

The global structure of the vesicle membrane is not due to chemical bonds between lipids but to hydrophobic effects which prevent them from escaping from the bilayer. In contrast, nothing stops the lipids from moving about and changing places with one another within the bilayer. Hence, the membrane behaves as a two dimensional fluid. On the other hand, the membrane is a structure which resists to stretching and bending and it can also be considered as a solid. Here, we propose to model the vesicle membrane as a degenerated elastic material. In the sequel, we will switch to an Eulerian formalism but we start from the more familiar Lagrangian description. We represent a piece of membrane as the result of the deformation of a reference domain $\Gamma_{\varepsilon}:=\Gamma \times(-\varepsilon, \varepsilon)$ where $\Gamma$ is a surface and $2 \varepsilon$ is the natural thickness of the membrane. The deformation and displacement of the membrane are given by a (one to one) mapping,

$$
\Phi: \Gamma_{\varepsilon} \longrightarrow \mathbf{R}^{3} \text {. }
$$

The free energy associated to such deformation reads

$$
\mathscr{F}(\Phi)=\int_{\Gamma_{\varepsilon}} f(D \Phi(x)) d x
$$

where $f: \mathbf{R}^{3 \times 3} \rightarrow \mathbf{R}_{+}$is the so called stored energy function. For simplicity, let us assume that $\Gamma \subset \mathbf{R}^{2}$ is a piece of a plane and that $f(I d)=0{ }^{1}$

Since, the membrane is a two dimensional fluid, the stored energy function has to be anisotropic. For an isotropic material, the symmetries of the problem are given by the relations $f(D \Phi(x) R)=f(D \Phi(x))$ for any $R \in \mathrm{SO}(3)$. Here, we expect that for any deformation $\Phi$ of the form

$$
\Phi(x)=\left(\Phi^{\prime}\left(x_{1}, x_{2}\right), x_{3}\right) \quad \text { with } \Phi^{\prime} \in C^{1}\left(\Gamma, \mathbf{R}^{2}\right) \text { volume preserving, }
$$

we have $f(D \Phi(x))=0$. We deduce that $f(D \Phi(x) A)=f(D \Phi(x))$ for any $A \in$ $\mathrm{SL}(3)$ such that $A \mathbf{e}_{3}=\mathbf{e}_{3}$ and $A \mathbf{e}_{3}^{\perp} \subset \mathbf{e}_{3}^{\perp}$. This leads to

$$
f(D \Phi(x))=g\left(\partial_{1} \Phi(x) \times \partial_{2} \Phi(x), \partial_{3} \Phi(x)\right),
$$

for some $g: \mathbf{R}^{3} \times \mathbf{R}^{3} \rightarrow \mathbf{R}$. Moreover,

$$
\partial_{1} \Phi(x) \times \partial_{2} \Phi(x)=\partial_{3} \Phi(x) \in S^{2} \quad \Longrightarrow \quad f(D \Phi(x))=0 .
$$

Notice that there exist smooth functions $f$ complying to the above symmetries for which equivalence holds - for instance $f(D \Phi(x))=\mid \partial_{1} \Phi(x) \times \partial_{2} \Phi(x)-$ $\left.\partial_{3} \Phi(x)\right|^{2}+\left(\left|\partial_{3} \Phi(x)\right|^{2}-1\right)^{2}$. The set of zero-energy deformations is larger than in the isotropic case. In this sense, we consider a highly anisotropic model.

Such stored energy functions have been proposed and studied by Pantz and Trabelsi in [11] where they perform a formal asymptotic analysis. With their notation, the present model corresponds to $\mathscr{W}_{0} \equiv 0$. It is fair to mention that the present study has been suggested by Olivier Pantz.

\footnotetext{
1 These restrictions rule out non-vanishing spontaneous curvatures but we will recover the general case in the Eulerian setting, at the end of the next subsection.
} 
Fig. 1.3 A tilt deformation.

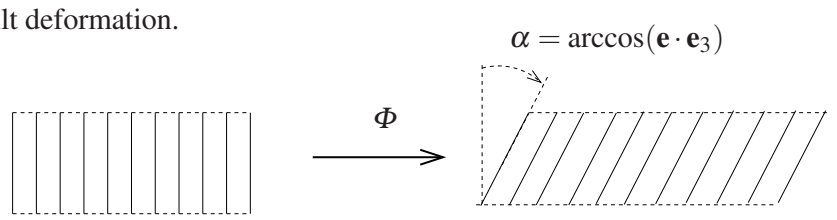

We have described the set of zero-energy deformations. Before switching to the Eulerian setting, let us briefly describe the simplest elements of the complement of this set: linear deformations which are penalized by an energy cost. In view of (1.5) the linear deformations which may cause an increase of the free energy are combinations of the form $\Phi=R \circ \Phi_{1} \circ \Phi_{2} \circ \Phi_{3}$ where $R$ is a rotation and at least one these elementary linear deformations $\Phi_{i}$ is not the identity. These elementary deformations are:

- a variation of the width of the membrane $\Phi_{1}\left(x^{\prime}, s\right)=\left(x^{\prime} / \sqrt{\lambda}, \lambda s\right)$ with $\lambda>0$,

- an isotropic variation of the density of the material, $\Phi_{2}(x)=\lambda x$,

- a tilt deformation of the form $\Phi_{3}\left(x^{\prime}, s\right)=\lambda x^{\prime}+s \mathbf{e}$, with $\lambda>0, \mathbf{e} \in S^{2}$ and $\operatorname{det} D \Phi_{3}=\lambda^{2} \mathbf{e} \cdot \mathbf{e}_{3}=1$ - see Figure 1.3.

\subsection{Eulerian version of the model}

In order to achieve a rigorous $\Gamma$-limit analysis, we have to establish some compactness results for sequences of deformations satisfying uniform energy bounds. For this task, the Lagrangian point of view is troublesome. Indeed, since any planar volume preserving rearrangement of the form (1.3) does not affect the energy, any compactness statement can only conclude to convergence up to right composition by such rearrangement. We avoid these difficulties by considering the Eulerian counterpart of the above model. This is also consistent with the fluid nature of the membrane.

Let us set $\Omega=\Phi\left(\Gamma_{\varepsilon}\right)$ and rewrite the energy as a function of $\Psi=\Phi^{-1}$. We obtain formally,

$$
\mathscr{F}_{E u}(\Psi, \Omega)=\int_{\Omega} f\left(D \Psi^{-1}(y)\right) \operatorname{det} D \Psi(y) d y=\int_{\Omega} f_{E u}(D \Psi) .
$$

The symmetry hypotheses on the Lagrangian model translate as follows:

$$
f_{E u}(A D \Psi(y))=f_{E u}(D \Psi(y))
$$

for any $A \in \operatorname{SL}$ (3) such that $A \mathbf{e}_{3}=\mathbf{e}_{3}$ and $A \mathbf{e}_{3}^{\perp} \subset \mathbf{e}_{3}^{\perp}$. The counterparts of (1.4) and (1.5) write,

$$
\begin{gathered}
f_{E u}(D \Psi(y))=g_{E u}\left(\nabla \Psi_{1}(y) \times \nabla \Psi_{2}(y), \nabla \Psi_{3}(y)\right), \\
\nabla \Psi_{1}(y) \times \nabla \Psi_{2}(y)=\nabla \Psi_{3}(y) \in S^{2} \Longrightarrow f_{E u}(D \Psi(y))=f_{E u}(I d) .
\end{gathered}
$$


Let us now get rid of the Lagrangian formalism. From the reverse deformation $\Psi$, we only need to keep track of the two following fields defined on $\Omega$,

$$
\sigma:=\nabla \Psi_{1} \times \nabla \Psi_{2} \text { and } t:=\Psi_{3} .
$$

By Piola identity, we know that $\nabla \cdot \sigma=0$ in $\Omega$. We also see that $|t|<\varepsilon$ in $\Omega$ and, if $\Phi$ is sufficiently smooth, that $t= \pm \varepsilon$ on $\partial \Omega$. Our model then rewrites formally as,

$$
\mathscr{F}_{E u}(\sigma, \tau, \Omega)=\int_{\Omega} g_{E u}(\sigma(y), \tau(y)) d y .
$$

where $\Omega$ is an open set, $\sigma$ is a divergence free vector field on $\Omega$ and where there exists $t \in C(\bar{\Omega},[-\varepsilon, \varepsilon])$ such that $\Omega=[|t|<\varepsilon]$ and $\tau=\nabla t$.

The quantities $\sigma$ and $t$ have natural physical meanings. We can see the reference shape $\Gamma \times(-\varepsilon, \varepsilon)$ as a collection of fibers with length $2 \varepsilon$, indexed by $x^{\prime} \in \Gamma$ and oriented by $\mathbf{e}_{3}$. Given $x^{\prime} \in \Gamma$, the corresponding reference fiber is described by $\left(x^{\prime}, s\right)$ where the abscissa $s$ on the fiber ranges from $-\varepsilon$ to $\varepsilon$. The deformation of this fiber is given by $\varphi_{x^{\prime}}(s)=\Phi\left(x^{\prime}, s\right)$. If we consider a single deformed fiber passing at some point $y \in \Omega$, then $t(y)$ gives the abscissa at this point on the corresponding reference fiber, that is the natural abscissa of this point on the fiber at rest. On the other hand, the relation $\Psi=\Phi^{-1}$ shows that $\sigma(y)=\nabla \Psi_{1} \times \nabla \Psi_{2}(y)$ is the flux of fibers passing at point $y$.

Therefore, $\sigma$ gives the local direction of the oriented fibers and $\tau=\nabla t$ is orthogonal to the level sets of the fiber natural abscissa. The relations on the left hand side of (1.6) mean that these directions are equal (no tilt), that the local elastic fiber is not stressed and that the local density of matter is the density at rest.

Eventually, we want to model closed membranes which separate the inside from the outside. To achieve this we assume that we can extend $t$ as a continuous function on $\mathbf{R}^{3}$ with values into $[-\varepsilon, \varepsilon]$. By convention, we assume $t \equiv \varepsilon$ in the neighborhood of infinity. The domain of the membrane is still $[|t|<\varepsilon]$ and the set $[t=-\varepsilon]$ defines the interior of the vesicle.

\subsection{Non-vanishing spontaneous curvature}

In order to take into account a non-vanishing spontaneous curvature $\mu$, we return to the Lagrangian description and endow the reference shape with a non-constant metric $m$. Namely, for $x \in \Gamma_{\varepsilon}$ and $\xi, \zeta \in T_{x} \Gamma_{\varepsilon} \simeq \mathbf{R}^{3}$, we set

$$
\langle\xi ; \zeta\rangle_{m(x)}:=\rho\left(\mu x_{3}\right) \xi^{\prime} \cdot \zeta^{\prime}+\xi_{3} \zeta_{3},
$$

where we note $\xi^{\prime}=\left(\xi_{1}, \xi_{2}\right)$ the planar components of a tangent vector and where $\rho: \mathbf{R} \rightarrow(0,+\infty)$ is a smooth function satisfying

$$
\rho(0)=1 \quad \text { and } \quad \rho^{\prime}(0)=-1 .
$$

The physical meaning of the metric is the following: given an open subset $\gamma \subset \Gamma$ and a height $t \in(-\varepsilon, \varepsilon)$, the quantity of rods which cross the oriented surface $\left(\gamma \times\{t\}, e_{3}\right)$ is $\rho(\mu t) \mathscr{H}^{2}(\gamma)$ and the "mass" of material inside a volume $\omega \subset \Gamma_{\varepsilon}$ is

$$
\int_{\omega} \rho\left(\mu x_{3}\right) d x
$$


Now, we consider a smooth deformation $\Phi: \Gamma_{\varepsilon} \rightarrow \mathbf{R}^{3}$. In the $m(x)$-orthonormal basis $\left(\rho^{-1 / 2}(x) e_{1}, \rho^{-1 / 2}(x) e_{2}, e_{3}\right)$ the components of its differential at $x$ write

$D_{m} \Phi(x):=M\left(x_{3}\right) D \Phi(x), \quad$ with $\quad M\left(x_{3}\right):=\operatorname{diag}\left(\frac{1}{\sqrt{\rho\left(\mu x_{3}\right)}}, \frac{1}{\sqrt{\rho\left(\mu x_{3}\right)}}, 1\right)$.

The energy associated with this deformation is

$$
\mathscr{F}(\Phi)=\int_{\Gamma_{\varepsilon}} f\left(D_{m} \Phi(x)\right) \rho\left(\mu x_{3}\right) d x
$$

The symmetry hypothesis (1.4) now reads,

$$
f\left(D_{m} \Phi(x)\right)=f\left(M\left(x_{3}\right) D \Phi(x)\right)=g\left(\left[1 / \rho\left(\mu x_{3}\right)\right] \partial_{1} \Phi(x) \times \partial_{2} \Phi(x), \partial_{3} \Phi(x)\right),
$$

and (1.5) is replaced by

$$
\left[1 / \rho\left(\mu x_{3}\right)\right] \partial_{1} \Phi(x) \times \partial_{2} \Phi(x)=\partial_{3} \Phi(x) \in S^{2} \quad \Longrightarrow \quad f\left(D_{m} \Phi(x)\right)=0 .
$$

Assuming that $\Phi$ is a smooth diffeomorphism onto its image $\Omega=\Phi\left(\Gamma_{\varepsilon}\right)$, we define $\Psi=\Phi^{-1}$ as above. In the Eulerian setting, the energy rewrites as

$$
\mathscr{F}(\Phi)=\mathscr{F}_{E u}(\Psi, \Omega):=\int_{\Omega} f_{E u}\left(\Psi_{3}(y), D \Psi(y)\right) d y
$$

with

$$
f_{E u}(t, A):=\rho(\mu t) \operatorname{det} A f\left(M(t) A^{-1}\right) \quad \text { for } t \in(-\varepsilon, \varepsilon) \text { and } A \in \mathrm{GL}_{3}(\mathbf{R}) .
$$

According to the symmetry hypothesis, the integrand rewrites as

$$
f_{E u}\left(\Psi_{3}, D \Psi\right)=\rho\left(\mu \Psi_{3}\right) \operatorname{det} D \Psi g\left(\frac{1}{\rho\left(\mu \Psi_{3}\right)}\left[\partial_{1} \Phi \times \partial_{2} \Phi\right] \circ \Psi,\left[\partial_{3} \Phi\right] \circ \Psi\right) .
$$

The identity $(\operatorname{Com} A)^{T}=(\operatorname{det} A) A^{-1}$ applied to $A=D \Psi$ and $A=D \Phi$ yields $\left[\partial_{3} \Phi\right] \circ \Psi=(\operatorname{det} D \Psi)^{-1} \nabla \Psi_{1} \times \nabla \Psi_{2}, \quad\left[\partial_{1} \Phi \times \partial_{2} \Phi\right] \circ \Psi=(\operatorname{det} D \Psi)^{-1} \nabla \Psi_{3}$.

We deduce that

$$
f_{E u}\left(\Psi_{3}, D \Psi\right)=\rho\left(\Psi_{3}\right) \operatorname{det} D \Psi g\left(\frac{\nabla \Psi_{3}}{\rho\left(\mu \Psi_{3}\right) \operatorname{det} D \Psi}, \frac{\nabla \Psi_{1} \times \nabla \Psi_{2}}{\operatorname{det} D \Psi}\right) .
$$

Now, let us define

$$
t:=\Psi_{3} \quad \text { and } \quad \sigma:=\rho(\mu t) \nabla \Psi_{1} \times \nabla \Psi_{2} .
$$

When $\mu \neq 0$, the definition of $\sigma$ is different from the definition of Section 1.2, here we substitute for the divergence free condition, the condition

$$
\nabla \cdot\left[(\rho(\mu t))^{-1} \sigma\right] \equiv 0 \quad \text { in } \Omega .
$$


With this notation (and since $\rho\left(\mu \Psi_{3}\right) \operatorname{det} D \Psi=\sigma \cdot \nabla t$ ) we obtain,

$$
f_{E u}\left(\Psi_{3}, D \Psi\right)=(\sigma \cdot \nabla t) g\left(\frac{1}{\sigma \cdot \nabla t} \nabla t, \frac{1}{\sigma \cdot \nabla t} \sigma\right)=: g_{E u}(\sigma, \nabla t)
$$

Notice that we still have the implication

$$
\sigma(y)=\nabla t(y) \in S^{2} \quad \Longrightarrow \quad g_{E u}(\sigma(y), \nabla t(y))=0
$$

As a conclusion, we have recovered the Eulerian model of Section 1.2 with the more general condition (1.7) on $\sigma$. To fix the ideas, in the sequel, we set $\rho(t):=$ $e^{-t}$ which corresponds to a constant growth of the "thickness" of the rods along their abscissa.

1.4 The general model in dimension $d$ and its zero energy configurations

Since it does not create any additional difficulty, we fix an integer $d \geq 2$ and set the problem in $\mathbf{R}^{d}$. The physical case corresponds to $d=3$.

Given $\mu \in \mathbf{R}$ and $\varepsilon>0$, a membrane of thickness $2 \varepsilon$ in $\mathbf{R}^{d}$ is modeled by a bounded open set $\Omega \subset \mathbf{R}^{d}$ and two mappings $\tau \in L^{2}\left(\mathbf{R}^{d}, \mathbf{R}^{d}\right)$ and $\sigma \in L^{2}\left(\Omega, \mathbf{R}^{d}\right)$. These objects are subjected to a set of constraints:

- there exists $t \in W_{l o c}^{1,2}\left(\mathbf{R}^{d}\right) \cap C\left(\mathbf{R}^{d},[-\varepsilon, \varepsilon]\right)$ such that $\tau=\nabla t$.

- $\Omega=\left\{y \in \mathbf{R}^{d}:|t|(y)<\varepsilon\right\}$.

- $\nabla \cdot\left[e^{\mu t} \sigma\right] \equiv 0$ in $\mathscr{D}^{\prime}(\Omega)$.

We will say that a configuration $a=(\sigma, \nabla t, \Omega)$ complying to these hypotheses is an $\varepsilon$-membrane.

The elastic energy associated to an $\varepsilon$-membrane has the form

$$
\mathscr{F}(a):=\int_{\Omega} f(\sigma(y), \nabla t(y)) d y
$$

where $f \in C\left(\mathbf{R}^{d} \times \mathbf{R}^{d}, \mathbf{R}_{+}\right)$depends on the material. In our context, the storedenergy functions $f$ of interest vanish on the sphere

$$
\mathbb{S}^{d-1}:=\left\{(e, e): e \in S^{d-1}\right\} \subset \mathbf{R}^{d} \times \mathbf{R}^{d}
$$

We assume that the stored energy function is not degenerated with respect to this property, that is $f(.) / d\left(\cdot, \mathbb{S}^{d-1}\right)^{2}$ is bounded from below by a positive constant on $\mathbf{R}^{d} \times \mathbf{R}^{d} \backslash \mathbb{S}^{d-1}$. 
1.5 Heuristic for thin vesicle membranes

For an $\varepsilon$-membrane $(\sigma, \nabla t, \Omega)$ with moderate energy, the vector fields $\nabla t$ and $\sigma$ should be close to one another, with magnitudes close to 1 . If we have exactly $(\sigma, \nabla t) \in \mathbb{S}^{d-1}$ then

$$
\Delta t=\nabla \cdot\left\{e^{-\mu t}\left[e^{\mu t} \sigma\right]\right\}=-\mu \nabla t \cdot \sigma=-\mu .
$$

We conclude that $\tau=\nabla t$ is a unit magnitude harmonic vector field in $\Omega$ for which the following Liouville type property holds.

Let $O \subset \mathbf{R}^{d}$, open. If $\tau: O \rightarrow S^{N}$ is harmonic, then $\tau$ is locally constant.

Proof (of (1.9)) Let $B \subset \bar{B} \subset O$ be an open ball with center $y$. Using $|\tau| \equiv 1$ and the mean value property, we compute,

$$
\oint_{B} \frac{|\tau-\tau(y)|^{2}}{2}=1-\tau(y) \cdot \oint_{B} \tau=1-|\tau(y)|^{2}=0
$$

and $\tau$ is constant in $B$.

In this zero energy case, the domain $\Omega=[|t|<\varepsilon]$ can only be a disjoint union of plates with width $2 \varepsilon$. It can not model a finite closed membrane: in our setting, membranes are always stressed.

Remark 1.1 Notice however that if we substitute another metric for $\rho(t)=e^{-\mu t}$ (but still assuming $\rho(0)=1$ and $\rho^{\prime}(0)=-\mu$ ) then (1.8) does not hold in general and zero-energy $\varepsilon$-membranes may exist. For instance, let $R>0$ and $\varepsilon \in(0, R)$ and let us set $\rho(t):=(1+t / R)^{d-1}$ — the spontaneous curvature is defined as $\mu:=-\rho^{\prime}(0)=-(d-1) / R$. In this setting their exists a zero-energy $\varepsilon$-membrane of domain $\Omega:=\left\{y \in \mathbf{R}^{d}: R-\varepsilon<|y|<R+\varepsilon\right\}$ with $\nabla t(y)=\sigma(y):=y /|y|$. A direct computation shows that $\nabla \cdot\left[\rho^{-1}(t) \sigma\right]$ vanishes in $\Omega$ as required.

To treat the case of a small but non-vanishing energy, we establish below rigidity inequalities which roughly state that if $(\sigma, \nabla t)$ is close to $\mathbb{S}^{d-1}$ in some domain, then $\sigma$ and $\nabla t$ are close to the same constant vector field in this domain. We expect that for configurations with a moderate energy, $\Omega$ is almost the $\varepsilon$-neighborhood of some close surface $\Sigma$ and that $\sigma$ and $\nabla t$ are close to the unit normal to $\Sigma$.

To get a more intuitive understanding of the link between our finite thickness model and Helfrich bending theory for surfaces, let us consider a fixed smooth hypersurface $\Sigma=\partial O \subset \mathbf{R}^{d}$ and let us build a family of $\varepsilon$-membranes with moderate energy and whose domain is the $\varepsilon$-neighborhood of $\Sigma$.

Let $\mu \in \mathbf{R}$ and let $O \subset \mathbf{R}^{d}$ be a smooth bounded open set, let $\Sigma=\partial O$ and $v$ be the outward unit normal on $\partial O$. We first define the function $t$ as the signed distance function from $\Sigma$.

$$
t(y):=d(y, O)-d\left(y, \mathbf{R}^{d} \backslash O\right) .
$$

Then for $\varepsilon>0$, we set $\Omega_{\varepsilon}=\{y:|t(y)|<\varepsilon\}$ and

$$
t_{\varepsilon}(y):= \begin{cases}t(y) & \text { if } y \in \Omega_{\varepsilon} \\ \pm \varepsilon & \text { if } \pm t(y) \geq \varepsilon .\end{cases}
$$


For $\varepsilon>0$ small enough the function $t_{\varepsilon}$ is smooth in $\Omega_{\varepsilon}$ and $\left|\nabla t_{\varepsilon}\right| \equiv 1$ in $\Omega_{\varepsilon}$. We then need to define a vector field $\sigma_{\varepsilon} \in L^{2}\left(\Omega_{\varepsilon}\right)$, close to $\nabla t_{\varepsilon}$ which satisfies $\nabla \cdot \sigma_{\varepsilon} \equiv \mu$ in $\mathscr{D}^{\prime}\left(\Omega_{\varepsilon}\right)$. For this, we consider the mapping

$$
\psi: \Sigma \times \mathbf{R} \rightarrow \mathbf{R}^{d}, \quad \psi(x, s):=x+s v(x) .
$$

There exists $\varepsilon_{\star}>0$ such that $\psi$ is a smooth difeomorphism from $\Sigma \times\left(-\varepsilon_{\star}, \varepsilon_{\star}\right)$ onto its image $\Omega_{\star}$. The inverse mapping is given by $\Psi^{-1}=(\pi, t)$ where $\pi$ is the orthogonal projection on $\Sigma$.

Let us now define the vector field $\sigma: \Omega_{\star} \rightarrow \mathbf{R}^{d}$ as

$$
\sigma(y):=\frac{e^{-\mu t(y)}}{\operatorname{det}\left[I d+t(y) \nabla_{\Sigma} v(\pi(y))\right]} \nabla t(y),
$$

We have to check that $e^{\mu t} \sigma$ is divergence free, that is

$$
Q(\varphi):=\int_{\Omega_{\star}} e^{-\mu t} \sigma \cdot \nabla \varphi=0 \quad \text { for every } \varphi \in \mathscr{D}\left(\Omega_{\star}\right) .
$$

Indeed, using the change of variable $y=\Psi(x, s)$ and noticing that the Jacobian determinant of $\Psi$ is $J_{\Psi}(x, s)=\operatorname{det}\left[\mathbf{I}_{d}+s \nabla_{\Sigma} v(x)\right]$, we get

$$
Q(\varphi):=\int_{\Sigma} \int_{-\varepsilon_{\star}}^{\varepsilon_{\star}} \frac{d}{d s}[\varphi(x+s v(x))] d s d \mathscr{H}^{d-1}(x)=0 .
$$

Substituting $y=\psi(x, s)$ in (1.10) and expanding the determinant with respect to $s$, we obtain

$$
\sigma(x+s v(x))=v(x)+s(h(x)-\mu) v(x)+O\left(s^{2}\right),
$$

where $h(x)=-\nabla_{\Sigma} \cdot v(x)$ is the scalar mean curvature on $\Sigma$.

Now, for $\varepsilon \in\left(0, \varepsilon_{\star}\right)$, we set

$$
\sigma_{\varepsilon}(y):= \begin{cases}\sigma(y) & \text { if } y \in \Omega_{\varepsilon}, \\ 0 & \text { if } y \in \mathbf{R}^{d} \backslash \Omega_{\varepsilon} .\end{cases}
$$

For $x \in \Sigma$ and $s \in(-\varepsilon, \varepsilon)$, we have $\nabla t_{\varepsilon}(x+s v(x))=v(x)$ and $\sigma_{\varepsilon}(x+s v(x))=$ $v(x)-s(h(x)-\mu) v(x)+O\left(s^{2}\right)$. Taking into account the identity $D f \equiv 0$ on $\mathbb{S}^{d-1}$, we see that the energy of $a_{\varepsilon}=\left(\sigma_{\varepsilon}, \nabla t_{\varepsilon}, \Omega_{\varepsilon}\right)$ expands as

$$
\mathscr{F}\left(a_{\varepsilon}\right)=\varepsilon^{3} \int_{\Sigma} c\left(D^{2} f(v, v)\right)(h-\mu)^{2} d \mathscr{H}^{d-1}+O\left(\varepsilon^{4}\right)
$$

where the function $c$ depends on the Hessian matrix of $f$ on $\mathbb{S}^{d-1}$.

In view of these computations, we expect that under symmetry hypotheses on $f$, the Helfrich energy (1.1) arises as the limit as $\varepsilon$ tends to 0 of the family $\left\{\mathscr{F} / \varepsilon^{3}\right\}$ defined on $\varepsilon$-membranes. This analysis is performed in the second part [10] of this paper in the case $\mu=0$ and under further hypotheses and volume constraints. In the language of $\Gamma$-convergence, we prove a compactness result, a lower bound result and we establish the matching upper bound in the smooth case. The corresponding analysis for the case $\mu \neq 0$ is also discussed in the concluding section of [10], albeit not completely detailed. 
1.6 The vanishing energy limit

From now on we assume $\mu=0$ and, in this first part, we consider the stored energy function $f=f_{0}$, with

$$
f_{0}(u, v):=|u-v|^{2}+(|u|-1)^{2}+(|v|-1)^{2} \quad \text { for every } u, v \in \mathbf{R}^{d} .
$$

Notice that $f_{0}(u, v) \sim\left[d\left((u, v), \mathbb{S}^{d-1}\right)\right]^{2}$ so this case illustrates non degenerate situations. The respective energy functional is denoted by

$$
\mathscr{F}_{0}(\sigma, \tau, \Omega):=\int_{\Omega} f_{0}(\sigma(y), \tau(y)) d y .
$$

As a first step toward the $\Gamma$-limit analysis of [10], we study the vanishing energy limit for configurations with fixed typical membrane width. When $\left(\sigma_{\varepsilon}, \nabla t_{\varepsilon}, \Omega_{\varepsilon}\right)$ is an $\varepsilon$-membrane, we can perform the scaling

$$
\Omega_{(\varepsilon)}:=\varepsilon^{-1} \Omega_{\varepsilon}, \quad t_{(\varepsilon)}(y):=\varepsilon^{-1} t_{\varepsilon}(\varepsilon y), \quad \sigma_{(\varepsilon)}(y):=\sigma_{\varepsilon}(\varepsilon y) .
$$

We easily see that $\left(\sigma_{(\varepsilon)}, \nabla t_{(\varepsilon)}, \Omega_{(\varepsilon)}\right)$ is a 1-membrane and

$$
\mathscr{F}_{0}\left(\sigma_{(\varepsilon)}, \tau_{(\varepsilon)}, \Omega_{(\varepsilon)}\right)=\varepsilon^{-d} \mathscr{F}_{0}\left(\sigma_{\varepsilon}, \tau_{\varepsilon}, \Omega_{\varepsilon}\right) .
$$

It is thus sufficient to consider membranes of width 2 . We prove the following compactness/structure result.

Theorem 1.1 Let $O \subset \mathbf{R}^{d}$ be a bounded open set.

Consider a sequence $a_{k}=\left(\sigma_{k}, \nabla t_{k}, O_{k}\right), k \geq 1$ such that

i) $O_{k} \subset O$ is open;

ii) $\sigma_{k} \in L^{2}\left(O_{k}, \mathbf{R}^{d}\right)$ is divergence free;

iii) $t_{k} \in W^{1,2}(O,[-1,1])$, satisfies $t_{k}= \pm 1$ in $O \backslash O_{k}$;

iv) $\left(t_{k}\right)$ is uniformly equi-continuous on $O$;

v) we have

$$
\mathscr{F}_{0}\left(\sigma_{k}, \nabla t_{k}, O_{k}\right) \rightarrow 0 \quad \text { as } k \uparrow \infty .
$$

Then, there exists $t_{\star} \in W^{1,2}(O) \cap C(\bar{O},[-1,1])$ such that, up to extraction,

$$
\begin{aligned}
t_{k} \rightarrow t_{\star} & \text { uniformly in } C(\bar{O}) \text {, weakly in } W^{1,2}(O), \\
& \text { and strongly in } W_{l o c}^{1,2}\left(O_{\star}\right) \text { with } O_{\star}:=\left\{y \in O:\left|t_{\star}\right|(y)<1\right\} .
\end{aligned}
$$

Moreover, $\nabla t_{\star}$ is locally constant with unit magnitude in $O_{\star}$.

More explicitly, the structure of the limit $t_{\star}$ is the following.

The function $t_{\star}$ is constant (equal to \pm 1 ) on any connected component of $O \backslash \overline{O_{\star}}$. For any connected component $\mathscr{C}$ of $O_{\star}$, there exist $e \in S^{d-1}$ and $y_{0} \in \mathbf{R}^{d}$ such that $t_{\star}(y)=\left(y-y_{0}\right) \cdot e$ for $y \in \mathscr{C}$. In particular $\mathscr{C}$ is a connected component of $O \cap\left\{y \in \mathbf{R}^{d}:\left|\left(y-y_{0}\right) \cdot e\right|<1\right\}$ (see Figure 1.4). Let us observe that this structure corresponds to a union of plane membranes with thickness 2 and with fibers aligned along the normal direction. 
Remark 1.2 Let us comment the equi-continuity assumption (iv) of the theorem. In general $(i v)$ is not a consequence of the uniform bound on the energy provided by $(v)$. However, by Morrey-Sobolev embedding, (iv) follows from the bound

$$
\sup _{k \geq 1} \int_{O}\left|\nabla t_{k}\right|^{p}<\infty \quad \text { for some } p>d \text {. }
$$

In particular, if we substitute for $\mathscr{F}_{0}$ the energy,

$$
\mathscr{F}(\sigma, \tau, \Omega):=\mathscr{F}_{0}(\sigma, \tau, \Omega)+\kappa \int_{\Omega}(|\nabla t|-1)^{p},
$$

for some $\kappa>0$ and $p>d$, then $(i v)$ follows from

$$
\mathscr{F}\left(\sigma_{k}, \nabla t_{k}, O_{k}\right) \rightarrow 0 \quad \text { as } k \uparrow \infty .
$$

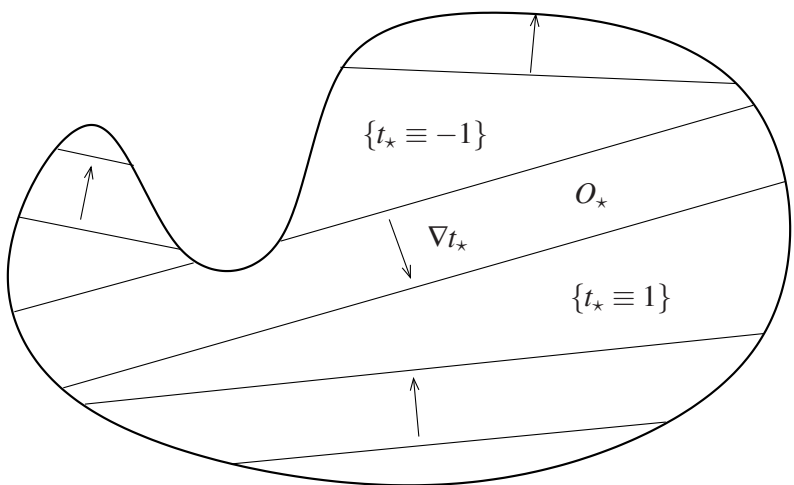

Fig. 1.4 Example of a vanishing energy limit state for which $O_{\star}$ has four connected components.

\subsection{Rigidity estimates}

The proof of Theorem 1.1 is based on some rigidity estimates. However the more precise rigidity estimates and their corollaries established in the present paper are not required for this task but are motivated by the $\Gamma$-convergence analysis exposed in the second part. In particular $\varepsilon-\delta$ type statements are not sufficient for this analysis and we need precise quantitative estimates.

For the compactness and lower bound results of [10], we are led to consider families $\left\{a_{\varepsilon}\right\}=\left\{\left(\sigma_{\varepsilon}, \nabla t_{\varepsilon}, \Omega_{\varepsilon}\right)\right\}_{0<\varepsilon \leq 1}$ such that $a_{\varepsilon}$ is an $\varepsilon$-membrane and satisfies the energy bound,

$$
\mathscr{F}_{0}\left(a_{\varepsilon}\right) \leq E_{0} \varepsilon^{3}
$$

We also require that the total mass of the membrane is $2 S \varepsilon$ for some fixed $S>0$,

$$
\int_{\Omega_{\varepsilon}} \sigma_{\varepsilon} \cdot \nabla t_{\varepsilon}=2 S \varepsilon .
$$


In the proof of the compactness result we build a smooth hypersurface $\Sigma_{\varepsilon}$ with total volume $\mathscr{H}^{d-1}\left(\Sigma_{\varepsilon}\right)=S+o(1)$. This hypersurface represents the membrane $\left(\sigma_{\varepsilon}, \tau_{\varepsilon}, \Omega_{\varepsilon}\right)$ in the sense that $\Omega_{\varepsilon}$ is close in $L^{1}$ to the $\varepsilon$-neighborhood of $\Sigma_{\varepsilon}$ and that the normal $v_{\varepsilon}$ on $\Sigma_{\varepsilon}$ is close to $\nabla t_{\varepsilon}$ and $\sigma_{\varepsilon}$. We then need to establish that the vector field $\nabla t_{\varepsilon}(y)$ is close in $\Omega_{\varepsilon}$ to the normal $v_{\varepsilon}(x)$ of a smooth surface passing through some point $x$ near $y$. This normal defines a "thickness" direction for the membrane. A natural candidate for such a direction is $\nabla t_{\varepsilon}$, this choice amounts to define $\Sigma_{\varepsilon}$ as a level set of $t_{\varepsilon}$. Since $\nabla t_{\varepsilon}$ may not be continuous, this choice is not reasonable without preparation: we first need to mollify $\nabla t_{\varepsilon}$. We are then led to consider averaged quantities and address the following issue:

Consider a closed ball $B_{\delta \varepsilon}(x) \subset \Omega_{\varepsilon}$, where $\delta>0$ is a small radius. Does their exist a direction $v_{\varepsilon}(x) \in S^{d-1}$ such that $\nabla t_{\varepsilon}$ is close to $v_{\varepsilon}(x)$ in $L^{2}\left(B_{\delta \varepsilon}(x)\right)$ ?

The relevant tool to tackle this problem is the energy bound (1.12). Since $\Omega_{\varepsilon}$ has volume $\mathscr{H}^{d-1}\left(\Omega_{\varepsilon}\right)=2 S \varepsilon+o(\varepsilon)$, this bound indicates that

$$
\frac{1}{\mathscr{H}^{d}\left(\Omega_{\varepsilon}\right)} \int_{\Omega_{\varepsilon}} f_{0}\left(\sigma_{\varepsilon}, \nabla t_{\varepsilon}\right)=O\left(\varepsilon^{2}\right) \text {. }
$$

Hence $f_{0}\left(\sigma_{\varepsilon}, \nabla t_{\varepsilon}\right)$ has typical order of $\varepsilon^{2}$. This implies that $\nabla t_{\varepsilon}$ is close to $S^{d-1}$ in $L^{2}\left(\Omega_{\varepsilon}\right)$ but also that $t_{\varepsilon}$ is almost harmonic in the following sense.

For every $\psi \in \mathscr{D}\left(B_{\delta \varepsilon}(x)\right)$, we have, since $\sigma_{\varepsilon}$ is divergence free:

$$
\int_{B_{\delta \varepsilon}(x)} \nabla t_{\varepsilon} \cdot \nabla \psi=\int_{B_{\delta \varepsilon}(x)}\left(\nabla t_{\varepsilon}-\sigma_{\varepsilon}\right) \cdot \nabla \psi \leq\left(\int_{B_{\delta \varepsilon}(x)} f_{0}\left(\sigma_{\varepsilon}, \nabla t_{\varepsilon}\right)\right)^{1 / 2}\|\nabla \psi\|_{L^{2}} .
$$

Hence, $\left\|\Delta t_{\varepsilon}\right\|_{H^{-1}\left(B_{\delta \varepsilon}(x)\right)}^{2} \leq \int_{B_{\delta \varepsilon}(x)} f_{0}\left(\sigma_{\varepsilon}, \tau_{\varepsilon}\right)=O\left(\varepsilon^{d+2}\right)$.

It is convenient to introduce the scaled function $\varphi(y):=\frac{1}{\varepsilon} t_{\varepsilon}(x+\varepsilon y)$. The preceding argument implies that the local energy

$$
\mathscr{E}:=\int_{B_{\delta}}(|\nabla \varphi|-1)^{2}+\|\Delta \varphi\|_{H^{-1}\left(B_{\delta}\right)}^{2}
$$

has typical order of $\varepsilon^{2}$.

In the limit case $\mathscr{E}=0, \nabla \varphi$ is a harmonic vector field with unit magnitude in $B_{\delta}$. The rigidity property (1.9) implies that $\nabla \varphi$ is constant in $B_{\delta}$. In this case, the natural normal direction is the constant unit vector $e=\nabla \varphi$.

For $\mathscr{E}>0, \mathscr{E} \sim \varepsilon^{2}$, we establish rigidity estimates associated to (1.9). The weakest form of these estimates states that:

$$
\forall \eta>0, \exists \beta>0 \text { such that } \mathscr{E}<\beta \Longrightarrow \min _{e \in S^{d-1}}\|\nabla \varphi-e\|_{L^{2}\left(B_{\delta}\right)}<\eta .
$$

The minimizer $e_{\star} \in S^{d-1}$ of $\|\nabla \varphi-e\|_{L^{2}\left(B_{\delta}\right)}$ then provides an average "thickness" direction.

Remark that the rigidity property (1.9) is related to the Liouville theorem: if $O \subset \mathbf{R}^{d}$ is an open set then for every $\psi \in W^{1,1}\left(O, \mathbf{R}^{d}\right)$,

$$
\nabla \psi \in \mathrm{SO}(d) \text { a.e. } \quad \Longrightarrow \quad \nabla \psi \text { is locally constant. }
$$


The associated $L^{2}$-rigidity estimate established by Frieseke, James and Müller in [6] is now classical: if $O \subset \mathbf{R}^{d}$ is a Lipschitz, bounded and connected open set, then,

$$
\inf _{R \in \operatorname{SO}(d)} \int_{O}|\nabla \psi-R|^{2} \leq C(O) \int_{O} d(\nabla \psi, \operatorname{SO}(d))^{2} \quad \text { for every } \psi \in W^{1,2}\left(O, \mathbf{R}^{d}\right) .
$$

In the same spirit, we establish some rigidity inequalities associated to (1.9).

It turns out that when we apply these rigidity estimates to a harmonic function $\varphi$, they provide some control on the curvature of the level sets of $\varphi$. In particular we can deduce bounds on their Willmore energy (1.2). These bounds are a key ingredient of the compactness step in [10]. Indeed, the main part of the hypersurface $\Sigma_{\varepsilon}$ is defined as a piece of a level set of some harmonic function. For this reason, the rigidity estimates stated in the present article are complemented by corollaries about level sets of harmonic functions.

\subsection{Notation}

Throughout the paper, the letter $C$ denotes a non negative constant which is either a universal constant or only depends on the dimension $d$. For constants which also depend on other parameters, $\alpha_{1}, \cdots, \alpha_{k}$, we write $C\left(\alpha_{1}, \cdots, \alpha_{k}\right)$. As usual, the values of these constants may change from line to line.

We write $B_{r}(y)$ to denote the open ball in $\mathbf{R}^{d}$ with center $y$ and radius $r>0$ or simply $B_{r}$ for $B_{r}(0)$.

The $k$-dimensional Hausdorff measure of a set $E \subset \mathbf{R}^{d}$ is denoted by $\mathscr{H}^{k}(E)$.

For $e \in S^{d-1}, \pi_{e}$ denotes the orthogonal projection on the space

$$
e^{\perp}=\left\{y \in \mathbf{R}^{d-1}: y \cdot e=0\right\},
$$

that is $\pi_{e}(y)=y-(y \cdot e) e$.

We also identify $e_{d}^{\perp}$ with $\mathbf{R}^{d-1}$ and for $y \in \mathbf{R}^{d}$ we write $y=\left(y^{\prime}, y_{d}\right)$ with $y^{\prime}=$ $\left(y_{1}, \cdots, y_{d-1}\right)=\pi_{e_{d}}(y)$.

\subsection{Outline of the first part}

In Section 2 we establish some local rigidity estimates associated to the Liouville property (1.9). We start with Theorem 2.1 which only applies to harmonic functions $\varphi$. This result will allow us to control the average Willmore energy of the level sets of such harmonic function (Corollary 2.1). Another application of Theorem 2.1 is the weak rigidity estimate (Theorem 2.2) which states that the $L^{2}$-distance from $\nabla \varphi$ to $S^{d-1}$ is controlled by the square root of the $L^{2}$-norm of $|\nabla \varphi|-1$. At the end of the section, we prove Theorem 1.1 as a consequence of this rigidity estimate.

In Section 3, we improve the rigidity estimates and obtain a linear control of the Willmore energy of the level sets of $\varphi$ with respect to the integral of $(|\nabla \varphi|-$ $1)^{2}$ (Corollary 3.1). 


\section{Weak rigidity estimates and proof of Theorem 1.1}

As stated above, if $O \subset \mathbf{R}^{d}$ is a non empty connected open set and if $\varphi: O \rightarrow \mathbf{R}^{d}$ is harmonic and satisfies $|\nabla \varphi| \equiv 1$ in $O$ then $\nabla \varphi$ is constant. We prove here various estimates associated to this rigidity property.

We begin by relaxing the constraint $|\nabla \varphi| \equiv 1$, still assuming that $\varphi$ is harmonic. As in [6], the first step relies on the Bochner idendity,

$$
\Delta\left[|\nabla \varphi|^{2}\right]=2 \nabla \varphi \cdot \nabla \Delta \varphi+2\left|D^{2} \varphi\right|^{2} \stackrel{\varphi \text { harm. }}{=} 2\left|D^{2} \varphi\right|^{2} .
$$

Theorem 2.1 (Weak rigidity estimate, harmonic case)

Let $O \subset \mathbf{R}^{d}$ be a nonempty open set and let $\varphi: O \rightarrow \mathbf{R}$ be a harmonic function. We assume moreover that $\varphi$ is nowhere locally constant, i.e. the interior of its critical set $\{y \in O ; \nabla \varphi(y)=0\}$ is empty.

Then, for $0 \leq \alpha<d /(d-1)$, the function $\left|D^{2} \varphi\right|^{2} /|\nabla \varphi|^{\alpha}$ is locally integrable and we have the estimate,

$$
\int_{O} \frac{\left|D^{2} \varphi\right|^{2}}{|\nabla \varphi|^{\alpha}} \chi \leq \frac{1}{1-\alpha(d-1) / d} \int_{O} \frac{|\nabla \varphi|^{2-\alpha}}{2-\alpha} \Delta \chi
$$

for every $\chi \in \mathscr{D}\left(O, \mathbf{R}_{+}\right)$.

\section{Remark 2.1}

$i$. The case $\alpha=0$ corresponds to Step 2 in the proof of Proposition 3.4 in [6].

ii. The proof combines the Bochner identity and the simple pointwise inequality (2.5). This line of reasoning is well known in the Geometric Analysis community (see e.g. [8], [13]). However, we did not find the estimate (2.1) in the literature. For example, it follows from [8]-Lemma 7.2 and [8]-Lemma 6.1 applied to $|\nabla \varphi|^{(d-2) /(d-1)}$ that for $0<\alpha<d /(d-1)$ and $O_{0} \subset \subset O$,

$$
\int_{O_{0}} \frac{\left.|\nabla| \nabla \varphi\right|^{2}}{|\nabla \varphi|^{\alpha}} \leq C\left(O_{0}, O, \alpha\right) \int_{O}|\nabla \varphi|^{2-\alpha} .
$$

In this inequality, the weight $\Delta \chi$ which appears in the right hand side of (2.1) is missing. This weight (or any other bounded weight with vanishing mean value) is necessary to establish Corollary 2.1.b below.

iii. An interesting consequence of the lemma is that for $\varphi$ harmonic in $O$ and $\beta:=1-\alpha / 2>(d-2) / 2(d-1)$, we have

$$
|\nabla \varphi|^{\beta} \in W_{l o c}^{1.2}(O) \text {. }
$$

In particular, in any dimension, $\sqrt{|\nabla \varphi|} \in W_{l o c}^{1,2}(O)$.

$i v$. The result does not hold in general if $\varphi$ is not harmonic, even if $\nabla \varphi$ is. For instance, if we choose $\varphi(y)=y_{1}^{2} / 2$, then

$$
\left|D^{2} \varphi\right|^{2} /|\nabla \varphi|^{\alpha}=1 /\left|y_{1}\right|^{\alpha} \notin L_{l o c}^{1}\left(\mathbf{R}^{d}\right) \text { for } \alpha \geq 1 .
$$

v. It transpires from the proof below that we have equality in (2.1) if for every $y \in O, \nabla \varphi(y)$ is an eigenvector of $D^{2} \varphi(y)$ associated to its eigenvalue with largest absolute value. Since this holds when $0 \notin O$ and $\varphi$ is proportional to the Green 
kernel of the Laplacian in $\mathbf{R}^{d}$, i.e. $\nabla \varphi(y)=c|y|^{-d} y$, we see that (2.1) is sharp. As shown by the counterexample $\varphi(y)=y_{1} y_{2}$, the conditions $\alpha<2, \beta>0$ for (2.2) and (2.3) are optimal in dimension $d=2$. In higher dimensions, we do not know the optimal exponents.

Proof (of Theorem 2.1) Let $O, \varphi$ and $\chi$ be as in the statement of the theorem, let $0 \leq \alpha \leq 2$ and let $\eta>0$ be a small parameter.

We start with the identity $\Delta\left[|\nabla \varphi|^{2} / 2\right]=\left|D^{2} \varphi\right|^{2}$ which holds for any harmonic function $\varphi$. Mutliplying this identity by $\chi /\left(\eta^{2}+|\nabla \varphi|^{2}\right)^{\alpha / 2}$ and integrating by parts, we get,

$$
-\int_{O} \frac{|\nabla \varphi|(\nabla|\nabla \varphi| \cdot \nabla \chi)}{\left(\eta^{2}+|\nabla \varphi|^{2}\right)^{\alpha / 2}}+\alpha \int_{O} \frac{\left|D^{2} \varphi \cdot \nabla \varphi\right|^{2}}{\left(\eta^{2}+|\nabla \varphi|^{2}\right)^{\frac{\alpha}{2}+1}} \chi=\int_{O} \frac{\left|D^{2} \varphi\right|^{2}}{\left(\eta^{2}+|\nabla \varphi|^{2}\right)^{\alpha / 2}} \chi .
$$

Using the notation,

$$
A(y):=D^{2} \varphi(y) \text { and } n_{\eta}(y):=\nabla \varphi(y) / \sqrt{\eta^{2}+|\nabla \varphi|^{2}(y)},
$$

the above identity rewrites as,

$$
\int_{O} \frac{|A|^{2}-\alpha\left|A n_{\eta}\right|^{2}}{\left(\eta^{2}+|\nabla \varphi|^{2}\right)^{\alpha / 2}} \chi=-\int_{O} \frac{|\nabla \varphi|}{\left(\eta^{2}+|\nabla \varphi|^{2}\right)^{\alpha / 2}} \nabla|\nabla \varphi| \cdot \nabla \chi
$$

We simplify the right hand side by using the identity,

$$
\nabla\left[\frac{\left(\eta^{2}+|\nabla \varphi|^{2}\right)^{1-\alpha / 2}}{2-\alpha}\right]=\frac{|\nabla \varphi|}{\left(\eta^{2}+|\nabla \varphi|^{2}\right)^{\alpha / 2}} \nabla|\nabla \varphi| .
$$

Integrating by parts, we get

$$
\int_{O} \frac{|A|^{2}-\alpha\left|A n_{\eta}\right|^{2}}{\left(\eta^{2}+|\nabla \varphi|^{2}\right)^{\alpha / 2}} \chi=\int_{O} \frac{\left(\eta^{2}+|\nabla \varphi|^{2}\right)^{1-\alpha / 2}}{2-\alpha} \Delta \chi
$$

Now, let us fix $y \in O$ and let us estimate from below the quantity $|A|^{2}(y)-$ $\alpha\left|A n_{\eta}\right|^{2}(y)$ which appears in the left hand side.

Since $\varphi$ is harmonic, the symmetric matrix $A(y)=D^{2} \varphi(y)$ has zero trace. Let $\lambda_{i}$, $i=1, \cdots, d$ be the eigenvalues of $A(y)$, sorted from least to greatest absolute value (in particular, $\left.\left|\lambda_{d}\right|=\max \left|\lambda_{i}\right|\right)$. Using $\left|n_{\eta}(y)\right| \leq 1$, we have,

$$
|A(y)|^{2}-\alpha\left|A(y) n_{\eta}(y)\right|^{2} \geq \sum \lambda_{i}^{2}-\alpha \lambda_{d}^{2}=|A(y)|^{2}\left(1-\alpha \beta_{d}^{2}\right),
$$

where we have set $\beta_{i}:=\lambda_{i} /|A(y)|$.

Minimizing the last term under the constraints $\sum_{i=1}^{d} \beta_{i}=0$ and $\sum_{i=1}^{d} \beta_{i}^{2}=1$, we obtain $\left(1-\alpha \beta_{d}^{2}\right) \geq(1-\alpha(d-1) / d)$. Hence, for $\alpha<d /(d-1)$,

$$
|A(y)|^{2}-\alpha\left|A(y) n_{\eta}(y)\right|^{2} \geq\left(1-\alpha \frac{d-1}{d}\right)|A(y)|^{2} .
$$


Assuming $\alpha<d /(d-1)$, and using this inequality in (2.4), we get,

$$
\left(1-\alpha \frac{d-1}{d}\right) \int_{O} \frac{\left|D^{2} \varphi\right|^{2} \chi}{\left(\eta^{2}+|\nabla \varphi|^{2}\right)^{\alpha / 2}} \leq \int_{O} \frac{\left(\eta^{2}+|\nabla \varphi|^{2}\right)^{1-\alpha / 2}}{2-\alpha} \Delta \chi
$$

Letting $\eta \downarrow 0$, we get by the monotone convergence theorem,

$$
\left(1-\alpha \frac{d-1}{d}\right) \int_{O} \frac{\left|D^{2} \varphi\right|^{2}}{|\nabla \varphi|^{\alpha}} \chi \leq \int_{O} \frac{|\nabla \varphi|^{2-\alpha}}{2-\alpha} \Delta \chi .
$$

This proves the theorem.

For our purpose, the interest of Theorem 2.1 resides in that it allows us to control the second fundamental form of the level sets of $\varphi$ in $L^{2}$.

Corollary 2.1 Let $O \subset \mathbf{R}^{d}$ be a connected, non empty open set and let $\varphi: O \rightarrow \mathbf{R}$ be a harmonic function.

Then, for almost every $s$, the set $\Gamma^{s}:=\varphi^{-1}(\{s\})$ is either empty or an analytic hypersurface. Moreover:

a) We have the following control on the $(d-1)$-volume of these hypersurfaces,

$$
\left|\int_{\mathbf{R}} \mathscr{H}^{d-1}\left(\Gamma^{s}\right) d s-\mathscr{H}^{d}(O)\right| \leq \int_{O}|| \nabla \varphi|-1| .
$$

b) Let $O_{0} \subset \subset O$ and let us set $\gamma^{s}:=\Gamma^{s} \cap O_{0}$ for $s \in \mathbf{R}$. We have the following control on the curvature of these level sets,

$$
\int_{\mathbf{R}}\left\{\int_{\gamma^{s}}\left|I_{s}\right|^{2}(y) d \mathscr{H}^{d-1}(y)\right\} d s \leq C\left(O_{0}, O\right) \int_{O}|| \nabla \varphi|-1|,
$$

where $I I_{S}$ denotes the second fundamental form on $\gamma^{s}$.

Remark 2.2 Thereafter, we improve the control on the Willmore energy of the level sets of $\varphi$ in domains where we already know that $|\nabla \varphi|$ is bounded from below by a positive constant. The strength of Theorem 2.1 and Corollary 2.1 lie in their robustness as they are valid in the neighborhood of the critical set $[\nabla \varphi=0]$.

Proof (of Corollary 2.1)

(a) By Sard theorem, for almost every $s$, the vector field $\nabla \varphi$ does not vanish on $\Gamma^{s}$. For such $s, \Gamma^{s}$ is either empty or an analytic hypersurface. Now, by the co-area formula, we have,

$$
\int_{O}|\nabla \varphi|=\int_{\mathbf{R}}\left[\int_{\Gamma^{s}} \mathbf{1}_{O} d \mathscr{H}^{d-1}\right] d s=\int_{\mathbf{R}} \mathscr{H}^{d-1}\left(\Gamma^{s}\right) d s .
$$

Writing $\int_{O}|\nabla \varphi|=\mathscr{H}^{d}(O)+\int_{O}[|\nabla \varphi|-1]$, we obtain the first estimate.

(b) Let us introduce a unit normal at the point $x \in \Gamma^{s}$ as $n(x):=\nabla \varphi /|\nabla \varphi|(x)$. Then, for $v, w \in n(x)^{\perp}$,

$$
I_{s}(x)(v, w)=-v^{T} \nabla n(x) w=-\frac{D^{2} \varphi(x)(v, w)}{|\nabla \varphi|(x)} .
$$


In particular, $\left|I_{s}\right|(x) \leq\left|D^{2} \varphi\right| /|\nabla \varphi|(x)$. Squaring and integrating on $\gamma_{s}$ and then in $s$, the co-area formula leads to,

$$
\int_{\mathbf{R}}\left\{\int_{\gamma^{s}}\left|I I_{s}\right|^{2} d \mathscr{H}^{d-1}\right\} d s \leq \int_{\mathbf{R}}\left\{\int_{\gamma^{s}} \frac{\left|D^{2} \varphi\right|^{2}}{|\nabla \varphi|^{2}} d \mathscr{H}^{d-1}\right\} d s=\int_{O_{0}} \frac{\left|D^{2} \varphi\right|^{2}}{|\nabla \varphi|} .
$$

Applying Theorem 2.1 with $\alpha=1$ and $\chi \in C_{c}^{\infty}\left(O, \mathbf{R}_{+}\right)$such that $\chi \geq \mathbf{1}_{\mathbf{O}_{0}}$, we get

$$
\int_{O_{0}} \frac{\left|D^{2} \varphi\right|^{2}}{|\nabla \varphi|} \leq d \int_{O}|\nabla \varphi| \Delta \chi=d \int_{O}(|\nabla \varphi|-1) \Delta \chi \leq C\left(O_{0}, O\right) \int_{O}|| \nabla \varphi|-1| .
$$

Combining the two last estimates leads to the desired result.

Now, we also relax the condition $\Delta \varphi \equiv 0$. In our context, the relevant quantity for measuring the distance from the constraints is the (local) energy,

$$
\mathscr{E}:=\int_{O}(|\nabla \varphi|-1)^{2}+\inf \left\{\int_{O}|\sigma-\nabla \varphi|^{2}: \sigma \in L^{2}(O)^{d}, \nabla \cdot \sigma \equiv 0\right\} .
$$

Notice that by definition of $\mathscr{F}_{0}$ (see (1.11)), we have $\mathscr{E} \leq \mathscr{F}_{0}(\nabla \varphi, \sigma, O)$ for any divergence free vector field $\sigma \in L^{2}(O)^{d}$.

When $O$ is bounded and $\nabla \varphi \in L^{2}(O)$, the infimum appearing in the right hand side of (2.6) is equal to $\|\Delta \varphi\|_{H^{-1}(O)}^{2}$. Indeed, the minimizer $\sigma$ satisfies the EulerLagrange equations

$$
\int(\sigma-\nabla \varphi) \cdot \hat{\sigma}=0, \quad \text { for every } \hat{\sigma} \in L^{2}(O)^{d} \text { s.t. } \nabla \cdot \hat{\sigma} \equiv 0 \text { in } \mathscr{D}^{\prime}(O) .
$$

By De Rham theorem, we see that $\sigma-\nabla \varphi=\nabla p$ where $p$ solves

$$
-\Delta p=\Delta \varphi \quad \text { in } \mathscr{D}^{\prime}(O), \quad p \in W_{0}^{1,2}(O) .
$$

The infimum in (2.6) is thus $\|\nabla p\|_{L^{2}(O)}^{2}=\|\Delta \varphi\|_{H^{-1}(O)}^{2} \leq \mathscr{E}$.

The notation (2.6) is used throughout this paper. We also use the decomposition $\varphi=\widetilde{\varphi}-p$ where $p$ solves (2.7). By construction, the function $\widetilde{\varphi} \in W^{1,2}(O)$ is harmonic and

$$
\int_{O}(|\nabla \widetilde{\varphi}|-1)^{2} \leq 2 \int_{O}(|\nabla \varphi|-1)^{2}+2 \int_{O}|\nabla p|^{2}=2 \mathscr{E} .
$$

We use Theorem 2.1 to control the distance (in $L^{2}(O)$ ) from $\nabla \varphi$ to the set of constant functions with values into $S^{d-1}$.

Theorem 2.2 (Weak rigidity estimate) Let $O \subset \mathbf{R}^{d}$ be a Lipschitz bounded and connected open set, then for every $\varphi \in W^{1,2}(O)$,

$$
\inf _{e \in S^{d-1}} \int_{O}|\nabla \varphi-e|^{2} \leq C(O)\left(\sqrt{\mathscr{H}^{d}(O) \mathscr{E}}+\mathscr{E}\right) .
$$


Remark 2.3 By homogeneity, the constant $K:=C(O)$ in (2.8) only depends on the shape of $O$, i.e. if $O^{\prime}=\lambda R O, \lambda>0, R \in \mathrm{O}(d)$ then (2.8) also holds in $O^{\prime}$ with $C\left(O^{\prime}\right)=K$.

Proof We split $\varphi$ in $\varphi=\widetilde{\varphi}-p$ as above. We have $\|\nabla \widetilde{\varphi}-\nabla \varphi\|_{L^{2}(O)}^{2} \leq \mathscr{E}$, so it is sufficient to establish the lemma for the harmonic function $\widetilde{\varphi}$. From now on we assume that $\varphi$ is harmonic.

Step 1 . Let us denote by $\eta$ the distance function $\eta:=d(\cdot, \partial O)$. In this step, we establish the following estimate.

$$
\int_{O}\left|D^{2} \varphi\right|^{2} \eta^{2} \leq\left. C \int_{O}|| \nabla \varphi\right|^{2}-1 \mid .
$$

Let us consider a partition $\mathscr{C}$ of $O$ in cubes of the form $K=y_{K}+\rho_{K}[-1,1)^{d}$ such that

$$
\begin{aligned}
\widetilde{K}:=y_{K}+\rho_{K}[-2,2)^{d} \subset O, \quad d\left(y_{K}, \mathbf{R}^{d} \backslash O\right) \leq 4 \operatorname{diam}(K) \\
\text { and for every } z \in O, \quad \#\{K \in \mathscr{C}: z \in \widetilde{K}\} \leq 2^{d} .
\end{aligned}
$$

To build such a partition, we start with a cube $K_{0} \supset O$ and subdivide it in $2^{d}$ halfcubes $K_{1}, \cdots K_{2^{d}}$. For every $i$, we check whether $\widetilde{K}_{i} \subset O$. If this is true, we pick $K_{i}$ (that is $K_{i} \in \mathscr{C}$ ), if not, we divide $K_{i}$ into $2^{d}$ equal subcubes $K_{i, 1}, \cdots, K_{i, 2^{d}}$ and proceed recursively.

Applying Theorem 2.1 in a cube $K=y+\rho[-1,1)^{d} \in \mathscr{C}$ with $\alpha=0$ and $\chi \in$ $\mathscr{D}(\widetilde{K})(y))$ such that $\chi \geq \mathbf{1}_{K}$ and $|\Delta \chi| \leq C / \rho^{2}$. We obtain,

$$
\rho^{2} \int_{K}\left|D^{2} \varphi\right| \leq\left. C \int_{\widetilde{K}}|| \nabla \varphi\right|^{2}-1 \mid
$$

By construction, for every $z \in K, d(z, \partial O) \leq 4 \sqrt{2 d} \rho_{K}$. Summing over all the elements of $\mathscr{C}$, we obtain (2.9).

Step 2 (Weighted Poincaré-Wirtinger inequality) Since $O$ is a Lipschitz, connected and bounded domain, the following weighted Poincaré inequality holds:

$$
\|\psi-\bar{\psi}\|_{L^{2}(O)}^{2} \leq C(O) \int_{O}|\nabla \psi|^{2} \eta^{2} \quad \text { for every } \psi \in W^{1,2}(O),
$$

where we use the notation $\bar{\psi}=\oint_{O} \psi$.

Let us establish this estimate for the convenience of the reader. For $\varepsilon>0$, we denote by $\partial_{\varepsilon} O$ the set

$$
\partial_{\varepsilon} O:=\{y \in \bar{O} ; d(y, \partial O) \leq \varepsilon\} .
$$

Let us first assume that there exist $\varepsilon>0$ and $\tau \in W^{1, \infty}\left(O, \mathbf{R}^{d}\right)$ such that

$$
O \backslash \partial_{\varepsilon} O \text { is connected, } \quad \nabla \cdot \tau \geq \frac{1}{2} \text { in } \partial_{\varepsilon} O, \quad \tau \equiv 0 \text { on } \partial O .
$$

Then, let $\psi \in W^{1,2}(O)$ and $m \in \mathbf{R}$, we compute

$$
\nabla \cdot\left\{(\psi-m)^{2} \tau\right\}=(\psi-m)^{2} \nabla \cdot \tau+2(\psi-m) \tau \cdot \nabla \psi .
$$


Integrating this identity on $O$, the left hand side vanishes since $\tau \equiv 0$ on $\partial O$. Using the (middle) inequality in (2.11) and the Cauchy-Schwarz inequality, we obtain,

$$
\frac{1}{2} \int_{\partial_{\varepsilon} O}(\psi-m)^{2} \leq\|\nabla \cdot \tau\|_{\infty} \int_{O \backslash \partial_{\varepsilon} O}(\psi-m)^{2}+2 \sqrt{\int_{O}(\psi-m)^{2}} \sqrt{\int_{O}|\tau|^{2}|\nabla \psi|^{2}}
$$

Using $2 a b \leq a^{2} / 4+4 b^{2}$ to bound the last term leads to

$$
\frac{1}{4} \int_{\partial_{\varepsilon} O}(\psi-m)^{2} \leq\left(\|\nabla \cdot \tau\|_{\infty}+\frac{1}{4}\right) \int_{O \backslash \partial_{\varepsilon} O}(\psi-m)^{2}+4 \int_{O}|\tau|^{2}|\nabla \psi|^{2} .
$$

Next, we set $m=\oint_{O} \backslash \partial_{\varepsilon} O \psi$ and we estimate the first term by applying the standard Poincaré-Wirtinger inequality in the (connected) open set $O \backslash \partial_{\varepsilon} O$. We get,

$$
\frac{1}{4} \int_{O}(\psi-m)^{2} \leq C\left(\tau, O \backslash \partial_{\varepsilon} O\right) \int_{O \backslash \partial_{\varepsilon} O}|\nabla \psi|^{2}+4 \int_{O}|\tau|^{2}|\nabla \psi|^{2}
$$

Eventually let us notice that $\eta /|\tau|$ is bounded from below in $O \backslash \partial_{\varepsilon} O$ by a positive number and that $|\tau| \leq\|\nabla \tau\|_{\infty} \eta$ (because $\tau \equiv 0$ on $\partial O$ ). We thus have,

$$
\int_{O}(\psi-m)^{2} \leq C(O) \int_{O} \eta^{2}|\nabla \psi|^{2}
$$

and since $m \mapsto \int_{O}(\psi-m)^{2}$ has its minimum at $\bar{\psi}$, we have established (2.10).

In order to conclude, we have to build $\tau \in W^{1, \infty}\left(O, \mathbf{R}^{d}\right)$ and $\varepsilon>0$ complying to (2.11). By hypothesis, we can cover $O$ with finitely many open subsets $Q_{0}, \cdots, Q_{N}$ such that $Q_{0} \subset \subset O$ and for $1 \leq k \leq N$, there exist $\rho_{k}>0$, a negative Lipschitz continuous function $f_{k}: B_{\rho_{k}} \cap \mathbf{R}^{\overline{d-1}} \rightarrow \mathbf{R}$ and an isometry of $\mathbf{R}^{d}$, $I_{k}(z)=I_{k}(0)+R_{k} z$, such that $Q_{k}=I_{k} \tilde{Q}_{k}$ with

$$
\tilde{Q}_{k}:=\left\{z \in \mathbf{R}^{d}: 0<\left|z^{\prime}\right|<\rho_{k}, f_{k}\left(z^{\prime}\right)<z_{d}<0\right\}
$$

and $y \in \overline{Q_{k}} \cap \partial O$ if and only if $z=I_{k}^{-1}(y)$ satisfies $\left|z^{\prime}\right| \leq \rho_{k}$ and $z_{d}=f_{k}\left(z^{\prime}\right)$.

Now, let $\chi_{0}, \cdots, \chi_{N}$ be a smooth partition of unity associated to $Q_{0}, \cdots Q_{N}$, we set

$$
\tau=\sum_{k=1}^{N} \chi_{k} \tau_{k}, \quad \text { with } \quad \tau_{k}\left(I_{k}(z)\right)=\left[z_{d}-f_{k}\left(z^{\prime}\right)\right] R_{k} e_{d} \text { for } z \in \tilde{Q}_{k} .
$$

It is a simple matter to check that (2.11) holds for $\varepsilon>0$ small enough.

Step 3 (Conclusion) The estimates (2.9) and (2.10) with $\psi=\nabla \varphi$ lead to

$$
\int_{O}|\nabla \varphi-\overline{\nabla \varphi}|^{2} \leq\left. C(O) \int_{O}|| \nabla \varphi\right|^{2}-1 \mid
$$

On the other hand, by the Cauchy-Schwarz inequality,

$$
\left.\int_{O}|| \nabla \varphi\right|^{2}-1\left|=\int_{O}\right||\nabla \varphi|-1||(|\nabla \varphi|-1+2) \leq \mathscr{E}+2 \sqrt{\mathscr{H}^{d}(O) \mathscr{E}} .
$$

Hence,

$$
\int_{O}|\nabla \varphi-\overline{\nabla \varphi}|^{2} \leq C(O)\left(\mathscr{E}+\sqrt{\mathscr{H}^{d}(O) \mathscr{E}}\right)
$$


Next, writing ||$\overline{\nabla \varphi}|-1| \leq|| \nabla \varphi|-1|+|\nabla \varphi-\overline{\nabla \varphi}|$, we also obtain,

$$
\mathscr{H}^{d}(O)(|\overline{\nabla \varphi}|-1)^{2} \leq C(O)\left(\mathscr{E}+\sqrt{\mathscr{H}^{d}(O) \mathscr{E}}\right)
$$

Considering separately the cases $|\overline{\nabla \varphi}| \leq 1 / 2$ and $|\overline{\nabla \varphi}|>1 / 2$, we deduce that there exists $e \in S^{d-1}$ such that

$$
\int_{O}|\nabla \varphi-e|^{2} \leq C(O)\left(\mathscr{E}+\sqrt{\mathscr{H}^{d}(O) \mathscr{E}}\right) .
$$

Indeed, in the case $|\overline{\nabla \varphi}| \leq 1 / 2$, the inequality is obvious for any $e \in S^{d-1}$. In the second case, it follows from (2.12) and (2.13) with $e:=\overline{\nabla \varphi} /|\overline{\nabla \varphi}|$. This proves the theorem.

To close the section, we establish Theorem 1.1. The main tool here is the weak rigidity inequality of Theorem 2.2 .

Proof (of Theorem 1.1)

Let $O$ and $\left(\sigma_{k}, t_{k}, O_{k}\right)$ be as in the statement of the theorem. By assumption the sequence $\left(t_{k}\right)$ is bounded in $W^{1,2}(O)$ and uniformly equi-continuous, so, up to extraction, there exists a (not relabeled) subsequence of $\left(t_{k}\right)$ weakly converging in $W^{1,2}(O)$ and strongly converging in $C(\bar{O})$ to some $t_{\star} \in W^{1,2}(O) \cap C(\bar{O},[-\varepsilon, \varepsilon])$.

Now, let $O_{\star}:=\left\{y \in O:\left|t_{\star}(y)\right|<\varepsilon\right\}$ and let $B \subset \subset O_{\star}$ be an open ball. By uniform convergence, there exists $k_{0} \geq 1$ such that $B \subset O_{k}$ for $k \geq k_{0}$. By definition of $\mathscr{E}$ (see (2.6) and the ensuing discussion) we have

$$
\mathscr{E}_{k}:=\int_{B}\left(\left|\nabla t_{k}\right|-1\right)^{2}+\left\|\Delta t_{k}\right\|_{H^{-1}(B)}^{2} \rightarrow 0 \quad \text { as } k \uparrow \infty .
$$

Applying Theorem 2.2 with $\varphi=t_{k}$ on the ball $B$, there exists $\left(v_{k}\right) \subset S^{d-1}$ such that,

$$
\left\|\nabla t_{k}-v_{k}\right\|_{L^{2}\left(B_{1}\right)} \stackrel{k \uparrow \infty}{\longrightarrow} 0 .
$$

Since $t_{k} \rightarrow t_{\star}$ in $\mathscr{D}^{\prime}(B)$, it follows that $\nabla t_{\star} \equiv e$ in $B$ for some $e \in S^{d-1}$.

\section{Strong rigidity estimates}

The weak rigidity estimate of Theorem 2.2 and Theorem 1.1 are used in the proof of the compactness result of [10] as $\varepsilon-\delta$ statements. They enable defining a local normal direction in the bulk of the membrane, at least away from a controlled number of balls of radius of order of $\varepsilon$. However, the sublinear growth of the right hand side of (2.8) with respect to the local energy $\mathscr{E}$ prevents deducing uniform $L^{2}$-bounds on the variation of this normal. For this, one could think about using an estimate of the form,

$$
\inf _{e \in S^{d-1}} \int_{O}|\nabla \varphi-e|^{2} \leq C(O) \mathscr{E} \quad \text { for every } \varphi \in W^{1,2}(O) .
$$


If $d=1$ and $O$ is a non empty interval, this estimate is obviously true. It also holds for $d=2$ when $O$ is a bounded and connected Lipschitz open set (see Remark 3.2) but not in higher dimensions.

Proof (Counterexample for (3.1) in the case $d \geq 3$ )

Let $\psi \in W^{1,2}(O)$ be harmonic. If (3.1) were true, writing $\varphi(y)=y_{d}+\eta \psi(y)$, and sending $\eta$ to 0 , we would obtain,

$$
\inf _{w \in e_{d}^{\perp}} \int_{O}|\nabla \psi-w|^{2} \leq C(O) \int_{O}\left|\partial_{d} \psi\right|^{2}
$$

If $\psi$ is a harmonic function that does not depend on $y_{d}$ and is not affine, the right hand side vanishes whereas the left hand side is positive. Since for $d \geq 3$, such functions do exist, this yields a contradiction.

Although (3.1) is too strong to be true, there is room for improving the estimate of Theorem 2.2 by lowering the left hand side. We find out a relevant correction by linearizing the constraints $|\nabla \varphi| \equiv 1$ and $\Delta \varphi \equiv 0$ around $\varphi_{0}(y)=e \cdot y$. Setting $\nabla \varphi=e+\nabla \psi$ we have at leading order, $e \cdot \nabla \psi \equiv 0$ and $\Delta \psi \equiv 0$. This suggests the following estimate.

$$
\inf _{\psi \in L_{O, e}} \int_{O}|\nabla \varphi-e-\nabla \psi|^{2} \leq C(O) \mathscr{E}
$$

where $e \in \operatorname{argmin}\left\{\int_{O}|\nabla \varphi-e|^{2}: e \in S^{d-1}\right\}$ and

$$
L_{O, e}:=\{\psi: O \rightarrow \mathbf{R}: \psi \text { is harmonic and } e \cdot \nabla \psi \equiv 0\} \text {. }
$$

Unfortunately, (3.2) is wrong in general.

Proof (Counterexample for (3.2))

Let us assume that $O$ is the unit ball in $\mathbf{R}^{3}$, let $p \geq 1$ be an integer and let us set (using cylindrical coordinates),

$$
\varphi(r, \theta, z):=z+\eta z r^{p} \sin p \theta
$$

By symmetry, the infimum in (3.2) is reached at point $e=e_{z}$ with $\psi \equiv 0$. Sending $\eta$ to $0,(3.2)$ leads to the contradiction $p^{2} \leq C$.

On the other hand, substituting a relatively compact convex subset $O_{0}$ for $O$ in the left hand side of (3.2)leads to a correct statement.

Theorem 3.1 Let $O$ be an open set, let $e \in S^{d-1}$ and let $O_{0} \subset \subset O$. Then, for every $\varphi \in W^{1,2}(O)$,

$$
\inf _{\psi \in L_{O_{0}, e}}\|\nabla \varphi-e-\nabla \psi\|_{L^{2}\left(O_{0}\right)}^{2} \leq C\left(O_{0}, O\right)\left(\mathscr{E}+\|\nabla \varphi-e\|_{L^{2}(O)}^{4}\right) .
$$


Remark $3.1 i$. The condition, $\psi \in L_{O_{0}, e}$ does not imply $\psi=\psi^{\prime} \circ \pi_{e}$ for some function $\psi^{\prime} \in W^{1,2}\left(\pi_{e}\left(O_{0}\right)\right)$. We obtain a counterexample in dimension 3 by considering the helix shape domains

$$
O_{0}:=\{(r \cos \theta, r \sin \theta, z): r \in(1 / 2,2), \theta \in(0,4 \pi),|z-\theta|<1 / 2\},
$$

and $O:=O_{0}+B_{1 / 4}$. The function defined on $O$ by

$$
\psi(r \cos \theta, r \sin \theta, z)=\sqrt{r} \cos (\theta / 2), \quad \text { with } \theta \text { such that }|\theta-z|<\pi
$$

is harmonic and we have $\partial_{3} \psi \equiv 0$ but since $\psi\left(y_{1}, y_{2}, z+2 \pi\right)=-\psi\left(y_{1}, y_{2}, z\right)$, we have $\left\|\xi^{\prime} \circ \pi_{e}-\nabla \psi\right\|_{L^{2}\left(O_{0}\right)} \geq \alpha>0$ for every vector field $\xi^{\prime} \in L^{2}\left(\pi_{e}\left(O_{0}\right), \mathbf{R}^{3}\right)$. Now, setting $\varphi_{\eta}=e_{3}+\eta \psi$ and sendig $\eta \downarrow 0$, we obtain

$$
\begin{aligned}
\inf _{\xi^{\prime}}\left\|\nabla \varphi_{\eta}-e_{3}-\xi^{\prime} \circ \pi_{e}\right\|_{L^{2}\left(O_{0}\right)}^{2} & \geq \alpha^{2} \eta^{2} \\
& \gg \quad\left\|\left|\nabla \varphi_{\eta}\right|-1\right\|_{L^{2}(O)}^{2}+\left\|\nabla \varphi_{\eta}-e_{3}\right\|_{L^{2}(O)}^{4}=O\left(\eta^{4}\right) .
\end{aligned}
$$

ii. However, if $O_{0}$ is convex or simply $e$-convex, then for $\psi \in L_{O_{0}, e}$ there exists a unique harmonic function $\psi^{\prime}$ on $\pi_{e}\left(O_{0}\right)$ such that $\psi=\psi^{\prime} \circ \pi_{e}$.

Proof (of Theorem 3.1.)

In view of Theorem 2.2, we can assume $\mathscr{E} \leq 1$. As above, we decompose $\varphi=$ $\widetilde{\varphi}-p$ with $\widetilde{\varphi}$ harmonic and $p \in W_{0}^{1,2}(O)$ such that $\|\nabla p\|_{L^{2}(O)}^{2} \leq \mathscr{E}$. We see that it is sufficient to establish (3.3) substituting $\widetilde{\varphi}$ for $\varphi$. Eventually, by isometry invariance, we can assume $e=e_{d}$.

From now on we assume $\mathscr{E} \leq 1, \varphi$ harmonic and $e=e_{d}$. To lighten notation, we set $Q:=\left\|\nabla \varphi-e_{d}\right\|_{L^{2}(O)}^{4}$.

Step 1 (finite cylinder case). Let us first assume that $O_{0}$ is a finite cylinder with direction $e_{d}$ and height 2: let $D^{\prime}$ be a smooth bounded open subset of $e_{d}^{\perp}=\mathbf{R}^{d-1}$, we assume that

$$
O_{0}=D:=\left\{y \in \mathbf{R}^{d}: y^{\prime} \in D^{\prime},-1<y_{d}<1\right\} .
$$

Let us also fix $\lambda>1$ such that $\lambda D \subset O$.

Let us write $\nabla \varphi=e_{d}+\nabla w$ and let us establish the inequality,

$$
\int_{\lambda D}\left|\partial_{d} w\right|^{2} \leq 2 \int_{\lambda D}(|\nabla \varphi|-1)^{2}+4 \int_{\lambda D}|\nabla w|^{4} \leq 4(\mathscr{E}+Q) .
$$

Let us fix $y \in \lambda D$ and let us set $a:=\partial_{d} w(y), b:=|\nabla w|^{2}(y) \geq a^{2}$. We start with the identity $|\nabla \varphi|(y)=\sqrt{1+2 a+b}$ so that

$$
a=(|\nabla \varphi|(y)-1)-(\sqrt{1+2 a+b}-1-a) .
$$

Assuming $a \geq-1 / 2$, we estimate the last term as

$$
|\sqrt{1+2 a+b}-1-a|=(1+a)\left(\sqrt{1+\frac{b-a^{2}}{(1+a)^{2}}}-1\right) \leq \frac{b-a^{2}}{2(1+a)} \leq b .
$$


Therefore, if $\partial_{d} w(y) \geq-1 / 2$, we have

$$
\left|\partial_{d} w\right|(y) \leq|| \nabla \varphi|(y)-1|+|\nabla w|^{2}(y)
$$

In the case $\partial_{d} w(y)<-1 / 2$, we use $\left|\partial_{d} w(y)\right| \leq 2|\nabla w|^{2}(y)$. Squaring and integrating these estimates over $\lambda D$, we obtain (3.5).

Now, let $\theta \in \mathscr{D}(-1,1)$ satisfying $\int \theta=1$ and let us define

$$
\psi_{\star}^{\prime}\left(y^{\prime}\right):=\int \theta(s) w\left(y^{\prime}, s\right) d s, \quad \text { for every } y^{\prime} \in \lambda D^{\prime} .
$$

By Poincaré-Wirtinger inequality, we have,

$$
\left\|w-\psi_{\star}^{\prime} \circ \pi_{e_{d}}\right\|_{L^{2}(\lambda D)}^{2} \leq C(\theta)\left\|\partial_{d} w\right\|_{L^{2}(\lambda D)}^{2} .
$$

Let us write $\psi^{\prime}\left(y^{\prime}\right)=\psi_{\star}^{\prime}\left(y^{\prime}\right)+\zeta^{\prime}\left(y^{\prime}\right)$ where $\zeta^{\prime} \in W_{0}^{1,2}\left(D^{\prime}\right)$ is the variational solution of

$$
-\Delta^{\prime} \zeta^{\prime}=\Delta^{\prime} \psi_{\star}^{\prime} \text { in } \lambda D^{\prime}, \quad \zeta^{\prime} \equiv 0 \text { on } \partial\left[\lambda D^{\prime}\right] .
$$

We compute,

$$
\Delta^{\prime} \zeta^{\prime}\left(y^{\prime}\right)=\int \theta(s) \Delta^{\prime} w\left(y^{\prime}, s\right) d s \stackrel{w}{=} \stackrel{\text { harm. }}{=}+\frac{d \theta p}{d s}(s) \partial_{d} w\left(y^{\prime}, s\right) d s .
$$

Therefore,

$$
\left\|\Delta^{\prime} \zeta^{\prime}\right\|_{L^{2}\left(\lambda D^{\prime}\right)}^{2} \leq C(\theta)\left\|\partial_{d} w\right\|_{L^{2}(\lambda D)}^{2} .
$$

We deduce from (3.6) and (3.7) that the harmonic function $\psi=\psi^{\prime} \circ \pi_{e_{d}}$ satisfies

$$
\|w-\psi\|_{L^{2}(\lambda D)}^{2} \leq C\left(D^{\prime}, \theta\right)\left\|\partial_{d} w\right\|_{L^{2}(\lambda D)}^{2} \stackrel{(3.5)}{\leq} C\left(D^{\prime}, \theta\right)(\mathscr{E}+Q) .
$$

By harmonic regularity, we conclude to:

$$
\left\|\nabla \varphi-e_{d}-\nabla \psi\right\|_{L^{2}(D)}^{2}=\|\nabla w-\nabla \psi\|_{L^{2}(D)}^{2} \leq C(D, O)(\mathscr{E}+Q) .
$$

This establishes the theorem, for $O_{0}$ of the form (3.4) and $e=e_{d}$.

Step 2 (stack of cylinders) Let us now assume that $O_{0}$ has the following form. Let $N \geq 1$ and $D_{1}^{\prime}, \cdots, D_{N}^{\prime}$ such that for every $0 \leq k \leq N, D_{k}^{\prime}$ is either empty or a smooth open subset of $\mathbf{R}^{d-1}$, we assume that

$$
O_{0} \text { is the interior of the set } \bigcup_{k=0}^{N} \overline{D_{k}} \quad \text { where } D_{k}:=D_{k}^{\prime} \times(k, k+1) \text {. }
$$

For $\alpha>0$, let us note $B_{\alpha}^{\prime}$ the $(d-1)$-ball $\left\{y^{\prime} \in \mathbf{R}^{d-1},\left|y^{\prime}\right|<\alpha\right\}$. By assumption, there exists $\alpha>0$ such that setting

$$
O_{0}^{b}:=\bigcup_{k=0}^{N} D_{k}^{b}, \quad \text { with } D_{k}^{b}:=D_{k}+\left[B_{\alpha}^{\prime} \times(-\alpha, \alpha)\right],
$$

we have $O_{0} \subset \subset O_{0}^{b} \subset \subset O$. 
Now, let $\varphi \in W^{1,2}(O)$. We first apply Step 1 in the sets $D_{k}^{b} \subset \subset O$. For every $0 \leq k \leq N$, there exist a harmonic function $\psi_{k}: D_{k}^{b} \rightarrow \mathbf{R}$ such that $\partial_{d} \psi_{k} \equiv 0$ and

$$
\left\|\nabla \varphi-e_{d}-\nabla \psi_{k}\right\|_{L^{2}\left(D_{k}^{b}\right)}^{2} \leq C\left(O_{0}, O\right)(\mathscr{E}+Q) .
$$

Notice that we can add any locally constant function to $\psi_{k}$ without altering the above properties. On this basis, we assume without loss of generality that

$$
\int_{X} \xi_{k}=0 \quad \text { on each connected component } X \text { of } D_{k}^{b}
$$

where $\xi_{k}: D_{k}^{b} \rightarrow \mathbf{R}$ is defined as $\xi_{k}(y):=\varphi(y)-y_{d}-\psi_{k}(y)$.

Let us also introduce for $0 \leq k \leq N$, the harmonic function $\psi_{k}^{\prime}: D_{k}^{b^{\prime}} \rightarrow \mathbf{R}$ such that $\psi_{k}=\psi_{k}^{\prime} \circ \pi_{e_{d}}$.

Let us now estimate the differences $\psi_{k}-\psi_{l}$ in $H^{1}$-norms. Let $0 \leq k \leq N-1$ such that $I_{k}^{\prime}:=D_{k}^{\prime} \cap D_{k+1}^{\prime} \neq \varnothing$. Using

$$
I_{k}:=\left[I_{k}^{\prime}+B_{\alpha}^{\prime}\right] \times(k+1-\alpha, k+1+\alpha) \subset D_{k}^{b} \cap D_{k+1}^{b}
$$

and (3.9) for $k$ and $k+1$, we first deduce:

$$
\begin{aligned}
\left\|\nabla \psi_{k}^{\prime}-\nabla \psi_{k+1}^{\prime}\right\|_{L^{2}\left(I_{k}^{\prime}+B_{\alpha}^{\prime}\right)} & =\frac{1}{\sqrt{2 \alpha}}\left\|\nabla \psi_{k}-\nabla \psi_{k+1}\right\|_{L^{2}\left(I_{k}\right)} \\
\leq & \frac{1}{\sqrt{2 \alpha}} \sum_{j \in\{k, k+1\}}\left\|\nabla \xi_{j}\right\|_{L^{2}\left(I_{k}\right)} \leq C\left(O_{0}, O\right) \sqrt{\mathscr{E}+Q} .
\end{aligned}
$$

Similarly, we compute, using (3.10) and the Poincaré-Wirtinger inequality,

$$
\begin{aligned}
\left\|\psi_{k}^{\prime}-\psi_{k+1}^{\prime}\right\|_{L^{2}\left(I_{k}^{\prime}+B_{\alpha}^{\prime}\right)} & \leq \frac{1}{\sqrt{2 \alpha}} \sum_{j \in\{k, k+1\}}\left\|\xi_{j}\right\|_{L^{2}\left(I_{k}\right)} \\
& \leq \sum_{j \in\{k, k+1\}} C\left(D_{j}^{b}\right)\left\|\nabla \xi_{j}\right\|_{L^{2}\left(D_{j}^{b}\right)} \stackrel{(3.9)}{\leq} C\left(O_{0}, O\right) \sqrt{\mathscr{E}+Q} .
\end{aligned}
$$

We summarize the two last inequalities as

$$
\left\|\psi_{k}^{\prime}-\psi_{k+1}^{\prime}\right\|_{W^{1,2}\left(I_{k}^{\prime}+B_{\alpha}^{\prime}\right)} \leq C\left(O_{0}, O\right) \sqrt{\mathscr{E}+Q} .
$$

Next, for $0 \leq k \leq l \leq N$, we note $J_{k, l}^{\prime}:=\cap_{k \leq j \leq l} D_{j}^{b^{\prime}}$. In particular, $J_{k, k+1}^{\prime}=I_{k}^{\prime}+B_{\alpha}^{\prime}$ for $k \leq N-1$, and $J_{k, l}^{\prime}$ is non-decreasing with respect to $k$ and non-increasing with respect to $l$.

Writing $\psi_{k}-\psi_{l}=\sum_{j=k}^{l-1}\left(\psi_{j+1}-\psi_{j}\right)$ and noticing that $J_{k, l}^{\prime} \subset I_{j}^{\prime}+B_{\alpha}^{\prime}$ for $k \leq j<l$, we deduce from (3.11),

$$
\left\|\psi_{l}^{\prime}-\psi_{k}^{\prime}\right\|_{W^{1,2}\left(J_{k, l}^{\prime}\right)} \leq C\left(O_{0}, O\right) \sqrt{\mathscr{E}+Q} .
$$


In order to define a global correction $\psi_{\star}(y)=\sum_{k=0}^{N} w_{k}(y) \psi_{k}^{\prime}\left(y^{\prime}\right)$, we need to build a partition of unity $w_{k}$ on $O_{0}$. For this, let us introduce a last sequence of $\left(e_{d}\right.$-convex) sets. For every $1 \leq k \leq N$, we define

$$
T_{k}:=\left[\bigcup_{l<k} J_{l, k}^{\prime} \times(l, k+1]\right] \cup D_{k}^{b^{\prime}} \times(0,1) \cup\left[\bigcup_{l>k} J_{k, l}^{\prime} \times[k, l+1)\right] .
$$

We now build a partition of unity with the required properties.

Claim There exist weight functions $w_{k} \in W^{1, \infty}\left(O_{0}, \mathbf{R}_{+}\right), k=0, \cdots, N$ such that $\sum_{k=0}^{N} w_{k} \equiv 1$ in $O_{0} \quad$ and for $0 \leq k \leq N, \quad \partial_{d} w_{k} \equiv 0$ in $O_{0} \quad$ and $\quad \operatorname{supp} w_{k} \subset T_{k}$.

Proof (of the claim) Let us introduce the relation $\sim$ on $O_{0}$ defined as $y \sim z$ if and only if $y \in z+\mathbf{R} e_{d}$ and $[y, z] \subset O_{0}$, or equivalently, if and only if $y$ and $z$ belong to the same connected component of $\left[z+\mathbf{R} e_{d}\right] \cap O_{0}$. For $y \in O_{0}$, we denote by $\bar{y}$ the class of $y$ in the quotient space $O_{0} / \sim$ and we denote by $P$ the canonical projection from $O_{0}$ onto $O_{0} / \sim$.

We define a distance on $O_{0} / \sim$. For this, we first set,

$d_{1}(\bar{y}, \bar{z}):= \begin{cases}\left|y^{\prime}-z^{\prime}\right| & \text { if there exists } \lambda \in \mathbf{R} \text { such that }\left(y^{\prime}, \lambda\right) \in \bar{y} \text { and }\left(z^{\prime}, \lambda\right) \in \bar{z} \\ +\infty & \text { in the other cases. }\end{cases}$

Notice that we have $d_{1}(\bar{y}, \bar{z}) \geq\left|y^{\prime}-z^{\prime}\right|$ for very $y^{\prime}, z^{\prime} \in O_{0}$. We then set,

$$
\begin{aligned}
& d(\bar{y}, \bar{z}):= \\
& \inf \left\{\sum_{i=0}^{R} d_{1}\left(\bar{y}_{i}, \bar{y}_{i+1}\right): R \geq 0, \bar{y}_{0}=\bar{y}, \bar{y}_{R+1}=\bar{z} \text { and } \bar{y}_{i} \in M \text { for } i=1, \cdots, R\right\} .
\end{aligned}
$$

We easily check that $d$ defines a distance with values into $[0,+\infty]$ on $O_{0} / \sim$. Moreover, we still have $d(\bar{y}, \bar{z}) \geq\left|y^{\prime}-z^{\prime}\right|$.

Notice also that for $E \subset O_{0} / \sim$, the mapping $d_{E}: y \in O_{0} \mapsto d(\bar{y}, E)$ is Lipschitzcontinuous on its domain $S=\left[d_{E}<\infty\right]$, with the bound $\left\|\nabla d_{E}\right\|_{L^{\infty}(S)} \leq 1$. Indeed, let $y \in O_{0}$ such that $d_{E}(y)<\infty$ and let $0 \leq k \leq N$ and $U$ be a neighborhood of $y$ such that $y \in U \subset D_{k}$. By definition of $d$, we have $d_{E}(z) \leq d_{E}(y)+|\bar{z}-\bar{y}|$ for every $z \in U$. By exchanging the roles of $y$ and $z$, we conclude that $\left|d_{E}(y)-d_{E}(z)\right| \leq$ $|\bar{y}-\bar{z}|$.

We are now ready to define the weight functions. We first set, for $0 \leq k \leq N$,

$$
\theta_{k}(y):=\max \left(0,1-\frac{d\left(\bar{y}, P\left(D_{k}\right)\right)}{2 \alpha}\right),
$$

so that $\theta_{k} \equiv 1$ on $D_{k},\left|\nabla \theta_{k}\right| \leq 1 /(2 \alpha)$ on $O_{0}$ and $\theta_{k}$ is constant on every segment of the form $y+(a, b) e_{d} \subset O_{0}$. Moreover, if $y \in \operatorname{supp} \theta_{k}$, then $d\left(\bar{y}, P\left(T_{k}\right)\right)<\alpha$ and by definition of $d$, there exists a finite chain $\bar{y}_{0}, \cdots, \bar{y}_{R+1} \in O_{0}$ with $\bar{y}_{0}=\bar{y}$ and $\bar{y}_{R+1} \subset D_{k}$ such that $\sum_{i} d_{1}\left(\bar{y}_{i}, \bar{y}_{i+1}\right)<\alpha$. By downward induction on $i$, we see that 
$\bar{y}_{i} \subset T_{k}$ for every $R+1 \geq i \geq 0$. In particular $\bar{y} \subset T_{k}$ and thus supp $\theta_{k} \subset T_{k}$.

Eventually, we define recursively,

$$
w_{0}:=\theta_{0} \quad \text { and } \quad w_{k}:=\left(1-\sum_{i<k} w_{i}\right) \theta_{k} \quad \text { for } k=1, \cdots, N .
$$

We easily check that the family $\left(w_{k}\right)$ complies to (3.13).

We can now define $\psi_{\star} \in W^{1,2}\left(O_{0}\right)$ as

$$
\psi_{\star}(y):=\sum_{k=0}^{N} w_{k}(y) \psi_{k}^{\prime}\left(y^{\prime}\right) \quad \text { for } y \in O_{0} .
$$

By construction, $\partial_{d} \psi_{\star} \equiv 0$ in $O_{0}$. In particular, for $1 \leq k \leq N$, the restriction of $\psi_{\star}(y)$ to $D_{k}$ does not depend on $y_{d}$. We note $\psi_{\star, k}^{\prime} \in W^{1,2}\left(D_{k}^{\prime}\right)$ the function such that $\psi_{\star}:=\psi_{\star, k}^{\prime} \circ \pi_{e_{d}}$ on $D_{k}$.

Using $\sum w_{l}=1$ and $\nabla w_{k}=-\sum_{l \neq k} \nabla w_{l}$, we obtain for $y \in D_{k}$,

$$
\nabla\left(\psi_{\star, k}-\psi_{k}\right)(y)=\sum_{l=1}^{N} w_{l}(y) \nabla\left(\psi_{l}^{\prime}-\psi_{k}^{\prime}\right)\left(y^{\prime}\right)+\sum_{l=1}^{N}\left(\psi_{l}-\psi_{k}\right)\left(y^{\prime}\right) \nabla w_{l}(y) .
$$

Taking into account (3.9), (3.12) and the fact that $\operatorname{supp} w_{l} \cap D_{k} \subset J_{k, l}$ for $k \leq l$ (respectively $J_{l, k}$ if $k>l$ ), we obtain:

$$
\left\|\nabla \varphi-e_{d}-\nabla \psi_{\star}\right\|_{L^{2}\left(O_{0}\right)}^{2} \leq C\left(O_{0}, O\right)(\mathscr{E}+Q) .
$$

Now let us set $\psi:=\psi_{\star}+\zeta$ where $\zeta \in W^{1,2}\left(O_{0}\right)$ is the variational solution to

$$
-\Delta \zeta=\Delta \psi_{\star} \text { in } O_{0}, \quad \zeta \equiv 0 \text { on } \Gamma, \quad \partial_{d} \zeta \equiv 0 \text { on } \Gamma^{\prime},
$$

where $\Gamma=\cup \partial D_{k}^{\prime} \times(k, k+1)$ denotes the vertical part of $\partial O_{0}$ and $\Gamma^{\prime}:=\partial O_{0} \backslash \Gamma$ is the horizontal part, i.e: $\Gamma^{\prime}=\partial O_{0} \cap\left[\cup_{k=1}^{N+1} \mathbf{R}^{d-1} \times\{k\}\right]$. In particular, the outward unit normal to $O_{0}$ on $\Gamma^{\prime}$ is $\pm e_{d}$, so that $\zeta$ satisfies homogeneous Neumann boundary condition on $\Gamma^{\prime}$.

The function $\psi$ is harmonic in $O_{0}$ and since $\partial_{d} \zeta$ solves $\Delta\left(\partial_{d} \zeta\right)=0$ in $O_{0}, \partial_{d} \zeta \equiv 0$ on $\partial O_{0}$, we have $\partial_{d} \zeta \equiv 0$ and thus $\partial_{d} \psi \equiv 0$. Eventually, we compute

$$
\begin{aligned}
& \|\nabla \zeta\|_{L^{2}\left(O_{0}\right)}^{2}=\int_{O_{0}} \nabla \psi_{\star} \cdot \nabla \zeta=\sum_{k=1}^{N} \int_{D_{k}} \nabla \psi_{\star} \cdot \nabla \zeta \\
& \stackrel{\psi_{k}^{\prime}}{=} \text { harm. } \sum_{k=1}^{N} \int_{D_{k}} \nabla\left(\psi_{\star}-\psi_{k}\right) \cdot \nabla \zeta \leq\left[\sum_{k=1}^{N}\left\|\nabla\left(\psi_{\star, k}^{\prime}-\psi_{k}^{\prime}\right)\right\|_{L^{2}\left(D_{k}^{\prime}\right)}\right]\|\nabla \zeta\|_{L^{2}\left(O_{0}\right)} \\
& \stackrel{(3.14)}{\leq} C\left(O_{0}, O\right) \sqrt{\mathscr{E}+Q}\|\nabla \zeta\|_{L^{2}\left(O_{0}\right)} .
\end{aligned}
$$

Hence, we can substitute $\psi$ for $\psi_{\star}$ in (3.14). This establishes the theorem for $O_{0}$ of the form (3.8) and $e=e_{d}$.

Step 3 (general case) 
Let $O_{0} \subset \subset O \subset \mathbf{R}^{d}$. For every direction $e^{b} \in S^{d-1}$, there exist a set $O_{0}^{b}$ of the form (3.8) and $O^{b} \subset \mathbf{R}^{d}$ such that $O_{0} \subset \subset O_{0}^{b} \subset \subset O^{b} \subset \subset O$, we can apply Step 2 to $O_{0}^{b} \subset O^{b}$ and deduce the theorem for $e=e^{b}$ and with a constant $C\left(e^{b}, O_{0}^{b}, O^{b}\right)$. The only remaining issue is that the constant in the right hand side of (3.3) should not depend on the particular direction $e$.

Now, for every $e^{b} \in S^{d-1}$, there exists a neighborhood $\mathscr{N}^{b}$ of $I d$ in $\operatorname{SO}(d)$ such that

$$
O_{0} \subset \subset R O_{0}^{b} \subset \subset R O^{b} \subset \subset O \quad \text { for every } R \in \mathscr{N}^{b} \text {. }
$$

By isometry invariance, the lemma also holds with the constant $C\left(e^{b}, O_{0}^{b}, O^{b}\right)$ for any direction $e \in \mathscr{N}^{b} e^{b}$. By compactness of $S^{d-1}$, we deduce that the lemma holds for any $e \in S^{d-1}$ with the constant $C\left(O_{0}, O\right):=\max _{e^{b} \in I} C\left(e^{b}, O_{0}^{b}, O^{b}\right)$ where $I$ is some finite subset of $S^{d-1}$ such that $\cup_{e^{b} \in I} \mathscr{N}^{b} e^{b}=S^{d-1}$.

Combining Theorem 2.2 and Theorem 3.1 we deduce the following result.

Theorem 3.2 (Strong rigidity inequality) Let $O$ be an open subset of $\mathbf{R}^{d}$ and let $O_{0} \subset \subset O$ be connected then for every $\varphi \in W^{1,2}(O)$ we have the estimate,

$$
\inf _{\psi \in L_{O_{0}, e_{\star}}} \int_{O_{0}}\left|\nabla \varphi-e_{\star}-\nabla \psi\right|^{2} \leq C(B, O) \mathscr{E}
$$

where $e_{\star} \in \operatorname{argmin}\left\{\int_{O}|\nabla \varphi-e|^{2}: e \in S^{d-1}\right\}$.

Remark 3.2 In dimension $d=2$ the set $L_{O_{0}, e_{\star}}$ reduces to the space of affine functions $\psi: O_{0} \rightarrow \mathbf{R}$ such that $\nabla \psi \equiv w$ for some $w \in e_{\star}^{\perp}$. By Theorem 2.2, we have $|w|^{2} \lesssim \sqrt{\mathscr{E}}$, so if we set $e:=\left(e_{\star}+w\right) /\left|e_{\star}+w\right|=e_{\star}+w+O(\sqrt{\mathscr{E}})$, Theorem 3.2 yields

$$
\int_{O_{0}}|\nabla \varphi-e|^{2} \leq C(B, O) \mathscr{E}
$$

Then, reasoning as in [6] (Proof of Theorem 3.1 from Proposition 3.4), we see that (3.2) holds in any connected and bounded Lipschitz domain.

When $\varphi$ is harmonic, Theorem 3.2 leads to an optimal control on the mean curvature of the level sets of $\varphi$. This result improves Corollary 2.1 in the sense that the right hand side is now linear in $\mathscr{E}$.

Corollary 3.1 Assume that $O$ is the non empty open ball $B_{r}(x)$. Let $\varphi: O \rightarrow \mathbf{R}$ be harmonic and let $\Gamma:=\{y \in O: \varphi(y)=\varphi(x)\}$.

There exists $\beta_{0}>0$ such that if $\mathscr{E} \leq \beta_{0} r^{d}$ then $\Gamma \cap B_{r / 2}(x)$ is an analytic hypersurface and we have the estimate,

$$
|I I|^{4}(x) \leq C r^{-(d+4)} \mathscr{E}, \quad|h|^{2}(x) \leq C r^{-(d+2)} \mathscr{E} .
$$

where II denotes the second fundamental form of $\Gamma$ and $h$ its mean curvature. 
Proof Using, the change of variable $\widetilde{\varphi}(y)=r^{-1}[\varphi(x+r y)-\varphi(x)]$, we see that we may assume that $O=B_{1}$ and $\varphi(0)=0$.

First, by Theorem 2.2 and harmonic regularity, we have,

$$
\left\|\nabla \varphi-e_{\star}\right\|_{L^{\infty}\left(B_{1 / 2}\right)}^{2} \leq C(\sqrt{\mathscr{E}}+\mathscr{E})
$$

with $e_{\star} \in \operatorname{argmin}\left\{\int_{B_{1}}|\nabla \varphi-e|^{2}: e \in S^{d-1}\right\}$. So, there exists $\beta_{0}>0$ such that if $\mathscr{E} \leq \beta_{0}$,

$$
\left\|\nabla \varphi-e_{\star}\right\|_{L^{\infty}\left(B_{1 / 2}\right)}^{2} \leq C \sqrt{\mathscr{E}} \leq 1 / 2 .
$$

Assuming, from now on, $\mathscr{E} \leq \beta_{0}$, we have $|\nabla \varphi| \geq 1 / 2$ in $B_{1 / 2}$ and the implicit function theorem implies that $\Gamma \cap B_{1 / 2}$ is an analytic hypersurface. In fact, it is easy to see that $\Gamma \cap B_{1 / 2}$ is a graph splitting $B_{1 / 2}$ into two topological balls.

Next, let us denote by $n(x)=\nabla \varphi /|\nabla \varphi|(x)$ the unit normal to $\Gamma$ at $x \in \Gamma \cap B_{1 / 2}$. We compute for $x \in \Gamma \cap B_{1 / 2}$, and $v, w \in n(x)^{\perp}$,

$$
v^{T} \nabla n(x) w=|\nabla \varphi|^{-1}(x) v^{T} D^{2} \varphi(x) w .
$$

Taking into account $|\nabla \varphi|(0) \geq 1 / 2$ and (3.16) we get by harmonic regularity,

$$
|I I|^{2}(0)=|\nabla n|^{2}(0) \leq 2\left|D^{2} \varphi\right|^{2}(0) \leq C \sqrt{\mathscr{E}}
$$

Let us now estimate the mean curvature $h(0)$. Solving the optimization problem which defines $e_{\star}$, we see that $e_{\star}=m /|m|$ with $m=\oint_{B_{1}} \nabla \varphi$. By the mean value property we have $m=\nabla \varphi(0)$, hence

$$
e_{\star}=n(0)
$$

Let us come back to (3.17). Taking the trace of $\nabla n(0)$ on $n(0)^{\perp}$ and using $\Delta \varphi=0$, we obtain,

$$
h(0)=-\nabla_{\Gamma} \cdot n(0)=|\nabla \varphi|^{-1}(0)\left[n^{T} D^{2} \varphi n\right](0) .
$$

By Theorem 3.2, there exists $\psi: B_{1 / 2} \rightarrow \mathbf{R}$, harmonic, such that $n(0) \cdot \nabla \psi \equiv 0$ and $\|\nabla \varphi-n(0)-\nabla \psi\|_{L^{2}\left(B_{1 / 2}\right)}^{2} \leq C \mathscr{E}$.

By harmonic regularity, we deduce $\left|D^{2}(\varphi-\psi)\right|(0) \leq C \sqrt{\mathscr{E}}$. In particular, since $D^{2} \psi(0) n(0)=0$, we have $\left|D^{2} \varphi n\right|(0) \leq C \sqrt{\mathscr{E}}$. Therefore,

$$
|h|^{2}(0) \leq 4\left|n^{T} D^{2} \varphi n\right|^{2}(0) \leq C \mathscr{E} .
$$

Unscaling (3.18) and this last estimate, we have established (3.15).

Acknowledgements The author wish to thank Olivier Pantz for suggesting this research subject and for inspiring discussions and Alberto Farina for pointing out the link between Theorem 2.1 and known results in Geometric Analysis. The author thanks the referee for his careful reading of the manuscript and his constructive comments. This research has been partially supported by the Agence National de la Recherche, through the project: ANR-12-BS01-0014-01 Geometrya. 


\section{References}

1. Sören Bartels, Georg Dolzmann, Ricardo H. Nochetto, and Alexander Raisch. Finite element methods for director fields on flexible surfaces. Interfaces Free Bound., 14(2):231$272,2012$.

2. Giovanni Bellettini and Luca Mugnai. Approximation of Helfrich's functional via diffuse interfaces. SIAM J. Math. Anal., 42(6):2402-2433, 2010.

3. Peter B. Canham. The minimum energy of bending as a possible explanation of the biconcave shape of the human red blood cell. J. Theor. Biol., 26(1):61-81, 1970.

4. Luca Deseri, Mario D. Piccioni, and Giuseppe Zurlo. Derivation of a new free energy for biological membranes. Contin. Mech. Thermodyn., 20(5):255-273, 2008.

5. Charles M. Elliott and Björn Stinner. Computation of two-phase biomembranes with phase dependent material parameters using surface finite elements. Commun. Comput. Phys. 13(2):325-360, 2013.

6. Gero Friesecke, Richard D. James, and Stefan Müller. A theorem on geometric rigidity and the derivation of nonlinear plate theory from three-dimensional elasticity. Comm. Pure Appl. Math., 55(11):1461-1506, 2002.

7. Wolfgang Helfrich. Elastic properties of lipid bilayers-theory and possible experimentsl. $Z$. Naturforsch, C28:693-703, 1973.

8. Peter Wai-Kwong Li. Harmonic functions and applications to complete manifolds. XIV Escola de Geometria Diferencial. [XIV School of Differential Geometry]. Instituto de Matemática Pura e Aplicada (IMPA), Rio de Janeiro, 2006.

9. Simon Masnou and Giacomo Nardi. Gradient young measures, varifolds, and a generalized willmore functional. Adv. Calc. Var., 2013.

10. Benoît Merlet. A highly anisotropic nonlinear elasticity model for vesicles. II: Derivation of the thin bilayer bending theory. Submitted, pages 1-57, 2013.

11. Olivier Pantz and Karim Trabelsi. A new non linear shell modeling combining flexural and membrane effects. to appear in, 2013.

12. Mark A. Peletier and Matthias Röger. Partial localization, lipid bilayers, and the elastica functional. Arch. Ration. Mech. Anal., 193(3):475-537, 2009.

13. Stefano Pigola, Marco Rigoli, and Alberto G. Setti. Vanishing and finiteness results in geometric analysis, volume 266 of Progress in Mathematics. Birkhäuser Verlag, Basel, 2008. A generalization of the Bochner technique.

14. Andreas Rätz and Matthias Röger. Turing instabilities in a mathematical model for signaling networks. J. Math. Biol., 65(6-7):1215-1244, 2012.

15. Brian Seguin and Eliot Fried. Microphysical derivation of the canham-helfrich free-energy density. ARXIV, pages $1-14,2012$.

16. Udo Seifert. Configurations of fluid membranes and vesicles. Adv. Phys., 46(1):13-173, 1997.

17. Matthew K. Turner and Jean-François Joanny. Diblock copolymer lamellae at rough surfaces. Macromolecules, 25(24):6681-6689, 1992. 